\title{
Host and viral determinants of influenza A virus species specificity
}

Jason S. Long ${ }^{1}$, Bhakti Mistry', Stuart M. Haslam² and Wendy S. Barclay ${ }^{1}$ *

Abstract | Influenza A viruses cause pandemics when they cross between species and an antigenically novel virus acquires the ability to infect and transmit between these new hosts. The timing of pandemics is currently unpredictable but depends on ecological and virological factors. The host range of an influenza A virus is determined by species-specific interactions between virus and host cell factors. These include the ability to bind and enter cells, to replicate the viral RNA genome within the host cell nucleus, to evade host restriction factors and innate immune responses and to transmit between individuals. In this Review, we examine the host barriers that influenza A viruses of animals, especially birds, must overcome to initiate a pandemic in humans and describe how, on crossing the species barrier, the virus mutates to establish new interactions with the human host. This knowledge is used to inform risk assessments for future pandemics and to identify virus-host interactions that could be targeted by novel intervention strategies.

\section{Zoonoses}

Diseases transmitted from animals to humans, such as H5N1 Avian influenza virus

Enzootic

Disease circulating in a non-human animal population.

Pandemic

The worldwide spread of a novel pathogen. Influenza pandemics occur when a new influenza virus emerges owing to a lack of immunity in the human population.

Seasonal influenza virus Human-adapted influenza viruses that regularly circulate and cause epidemics in the human population.

Glycans

Carbohydrates or assemblies of carbohydrates that are attached to proteins or lipids.

${ }^{1}$ Department of Medicine, Imperial College London, London, UK.

${ }^{2}$ Department of Life Sciences, Imperial College London, London, UK.

*e-mail:w.barclay@ imperial.ac.uk
Influenza viruses comprise a family of four distinct viruses: influenza $A, B, C$ and $D$ viruses. Influenza $A$ viruses infect a wide range of host species, whereas the main hosts for influenza B and C are humans, and influenza D virus has thus far been found to infect cattle, goats and pigs ${ }^{1}$ (FIG. 1). Influenza A virus is a paradigm for an emerging virus. A large and antigenically diverse reservoir of influenza A viruses exists in wild aquatic birds, in which the infection is usually asymptomatic ${ }^{2}$. Avian influenza viruses (AIVs) can cross into new species. For example, AIVs have transmitted from ducks to chickens or pigs and from birds to seals. Two antigenically distinct subtypes of influenza virus have also been detected in bats, but bat influenza viruses have not been observed to cross into other species ${ }^{3}$. Occasionally, influenza viruses of animals infect humans. Many of these zoonoses are dead ends (that is, there is no subsequent transmission) and occur in only a small number of individuals. Newly acquired viruses become enzootic in a new species only after genetic changes accumulate that adapt the virus to its new host and support efficient replication and transmission. When a novel influenza virus first accumulates sufficient adaptive mutations to sustain transmission between humans, it may result in a pandemic. During the first pandemic waves, a large proportion of the human population is infected and acquires immunity to the novel virus, but antigenically drifted variants of the pandemic virus emerge and continue to circulate as seasonal influenza virus ${ }^{4}$ (BOX 1).

Influenza viruses that are enzootic in animals can be experimentally studied in their natural host species (for example, laboratory infections of chickens or pigs).
Mice, guinea pigs and ferrets are the primary animal models for human influenza infection, important for studying pathogenesis, evaluating interventions and understanding mammalian adaptation requirements of avian viruses (reviewed in REF. ${ }^{5}$ ). Ferrets are considered the 'gold standard' for assessing the ability of influenza viruses to transmit via respiratory droplets, one of several parameters that are used to assess the pandemic potential of emerging influenza viruses (reviewed in $\mathrm{REFS}^{6,7}$ ).

Influenza viruses rely on numerous host factors to support their replication. Conversely, restrictive host factors can control or curtail infections. The drivers of host-adaptive evolution are differences in identity and/or expression patterns of these host factors in different species. This Review summarizes the latest information about the role of host factors during the influenza virus replication cycle, differences between species and how influenza viruses adapt to these differences.

\section{Species specificity of virus attachment}

The first host species barrier is host cell attachment. Influenza virus haemagglutinin (HA) binds to sialic acid (SA) moieties that are the terminal sugars linked to the larger glycans of glycoproteins and glycolipids (glycoconjugates) on the surface of vertebrate cells. Differences in the structure of these sialylated glycoconjugates between species can determine species-specific susceptibility to influenza virus infection.

Sialylation of host glycoconjugates. SAs are a diverse family of negatively charged monosaccharides that possess a common nine-carbon backbone. 


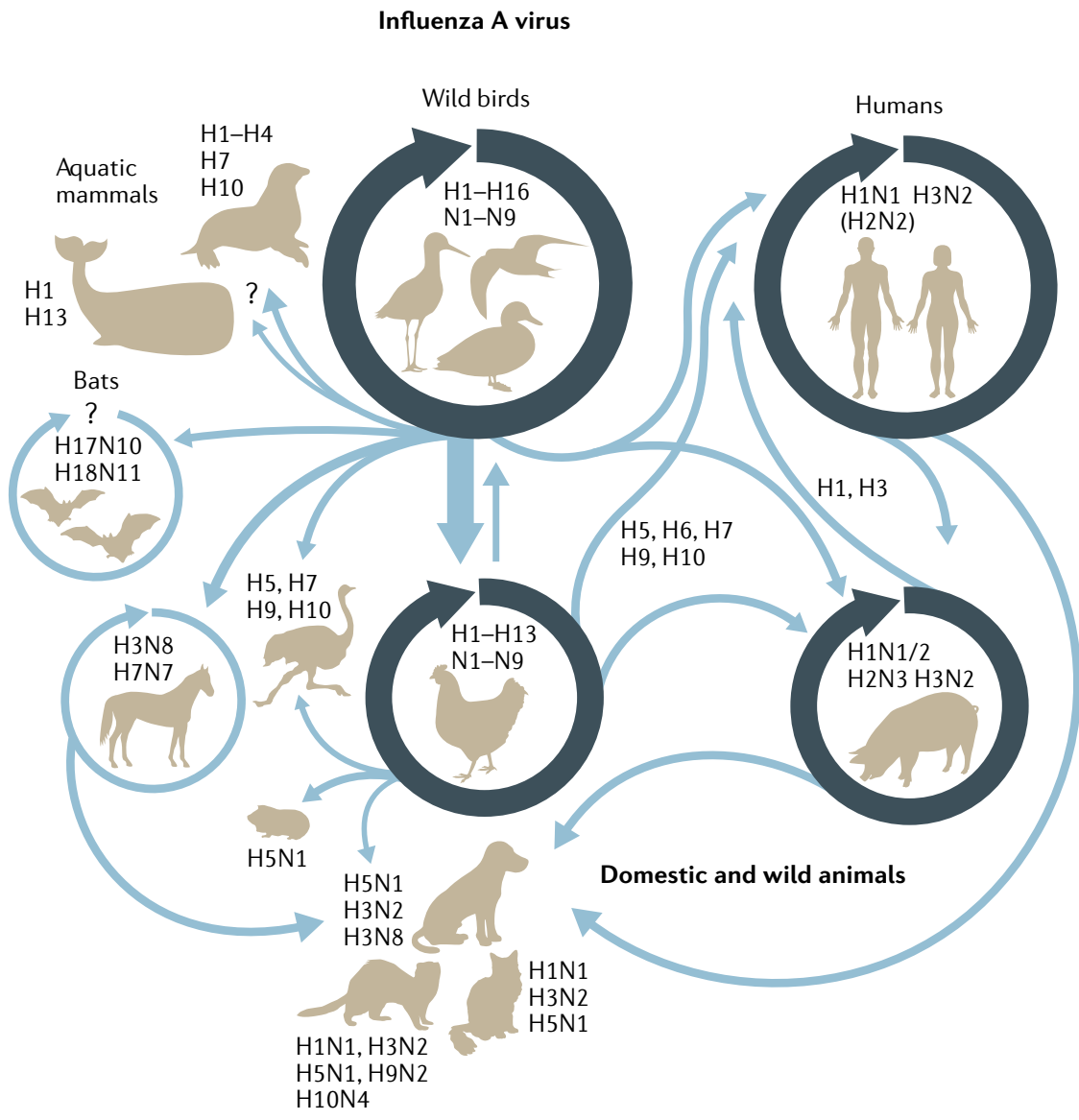

Influenza B virus

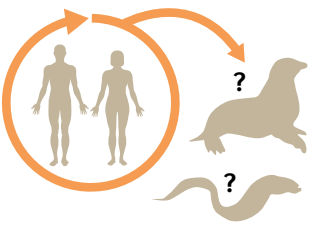

Influenza $\mathrm{C}$ virus

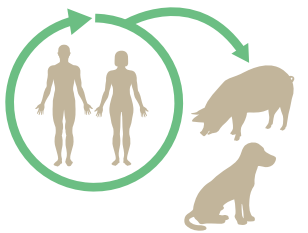

Influenza D virus

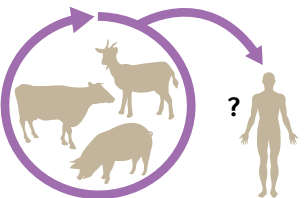

Other influenza viruses

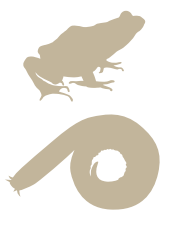

Experimental animals

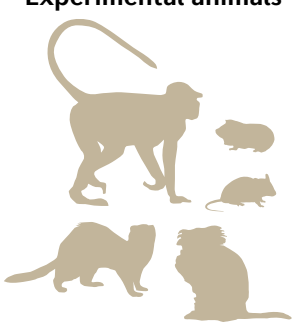

Fig. 1 | Ecology of influenza viruses. Influenza A viruses (haemagglutinin (HA) subtypes 1-16) circulate in the wild bird reservoir. Subtypes from this reservoir are able to cross into many different species, sometimes via intermediate hosts and sometimes requiring adaptive mutations (light blue arrows). Specific subtypes predominate in certain species (dark blue circles). Human-adapted influenza viruses of the $\mathrm{H} 1, \mathrm{H} 2$ and $\mathrm{H} 3$ subtypes have circulated in recent history. $\mathrm{H} 1 \mathrm{~N} 1$ and $\mathrm{H} 3 \mathrm{~N} 2$ viruses currently circulate whereas $\mathrm{H} 2 \mathrm{~N} 2$ viruses do not; the same three subtypes have also circulated in pigs. Avian influenza viruses (AIVs) of $\mathrm{H} 5, \mathrm{H} 6, \mathrm{H} 7, \mathrm{H} 9$ and $\mathrm{H} 10$ subtypes have infected humans following exposure to infected poultry. Viruses of these subtypes currently do not transmit between humans. HA subtypes $\mathrm{H} 17$ and $\mathrm{H} 18$ circulate only in bats ${ }^{169}$. Influenza B viruses circulate in humans, although infections in seals have been described ${ }^{170}$. Influenza $\mathrm{C}$ viruses circulate in humans and swine ${ }^{171}$. The recently discovered influenza $D$ viruses are found to circulate in cattle, goats and pigs ${ }^{1}$. Despite some serological evidence of infection in humans, the zoonotic threat to humans remains unclear. Detection of influenza virus-like RNA in Wuhan Asiatic toads, Wenling hagfish and spiny eels has recently been described, although their genera remain to be defined ${ }^{172}$. Many species have been experimentally infected by influenza viruses, including ferrets, mice, guinea pigs, macaques and marmosets (reviewed in $\mathrm{REFS}^{6,7}$ ).

There is considerable variation in the $\sim 60$ genes that encode SA biosynthetic pathways in different species. The majority of mammalian and avian species present two main types of SA on the terminal positions of the glycans of their glycoproteins and glycolipids $-\mathrm{N}$-acetylneuraminic acid (Neu5Ac) and $\mathrm{N}$-glycolylneuraminic acid (Neu5Gc). Neu5Gc is synthesized from Neu5Ac through the action of the enzyme CMP-Neu5Ac hydroxylase (CMAH). As the CMAH gene in humans is non-functional, Neu5Gc is not synthesized. This non-functional gene was likely selected for in ancient hominids because it conferred some resistance and differential immune responses to infection by pathogens ${ }^{8-10}$. Ferrets also have a non-functional CMAH and do not express Neu5Gc ${ }^{11}$. The differential expression of SAs in different species contributes to influenza virus species specificity. Indeed, equine influenza viruses preferentially bind Neu5Gc-containing glycoconjugates, whereas human influenza virus receptors preferentially bind Neu5 $\mathrm{Ac}^{11,12}$.

Sialic acid as a determinant of host range. For over 30 years, a central dogma of influenza virus research has been that the linkage of the terminal SA moiety to the subterminal galactose of glycans is fundamental to species specificity. HAs from human-adapted viruses bind a2-6-linked SA whereas AIV HAs bind a2-3-linked SA ${ }^{13}$ (FIG. 2). Consequently, human influenza viruses possess adaptive mutations in the receptor binding site (RBS) of HA compared with AIVs ${ }^{14}$. The explanation for the differential binding has been obtained from numerous X-ray crystallographic and glycan microarray binding studies. It has been proposed that in the RBS 


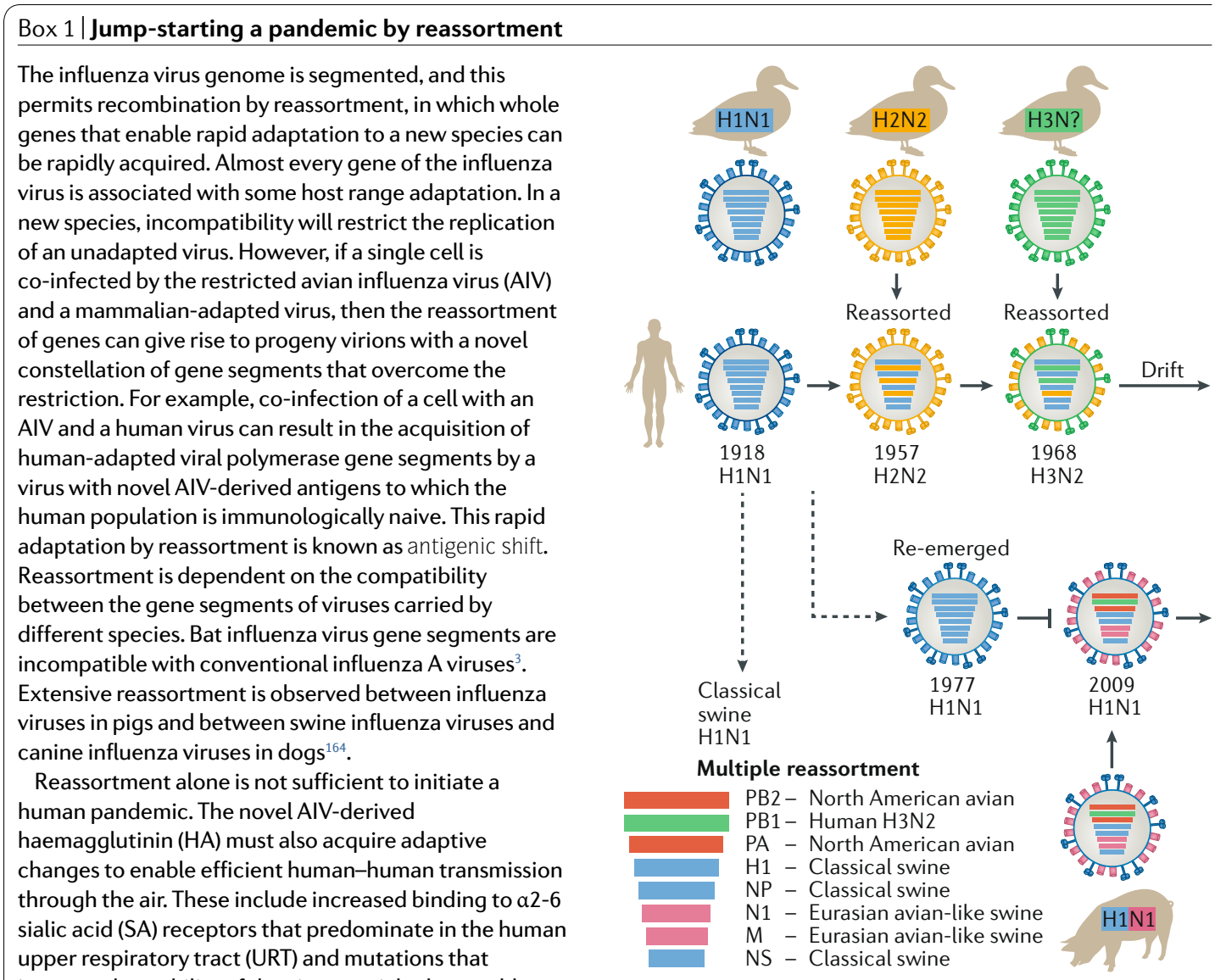
increase the stability of the virus particle that enable survival in airborne droplets and at the respiratory mucosa. This evolution occurs through the accumulation of mutations by the error-prone viral polymerase and the selection of viruses that are better able to replicate and transmit.

Influenza A viruses have thus far caused five pandemics in the 20th and 21st centuries (see the figure). The origin of the 1918 pandemic virus is controversial, but some sources suggest a recent avian origin of all eight RNA segments ${ }^{165}$. After the first few pandemic waves, newly emerged influenza viruses continue to circulate as seasonal influenza undergoing antigenic drift, in which the error-prone viral polymerase results in mutations that are selected to evade accumulating immune responses in the exposed population. Reassortment between 1918 virus derivatives and AIVs resulted in Asian influenza pandemic H2N2 virus in 1957 and Hong Kong influenza pandemic H3N2 virus in 1968. The colours of the eight gene segments (polymerase basic proteins 1 (PB1) and 2 (PB2), polymerase acidic protein (PA), HA, nucleoprotein (NP), neuraminidase (NA), matrix (M) and non-structural protein (NS)) describe their origins. Each of these pandemic viruses displaced the previously circulating human seasonal virus. In 1977, a Russian influenza pandemic H1N1 virus emerged with remarkable genetic similarity to H1N1 viruses that had circulated in humans in the early 1950s. The $1977 \mathrm{H} 1 \mathrm{~N} 1$ virus did not displace the seasonal H3N2 virus, which continued to circulate. In 2009, an H1N1 virus emerged from swine. This virus was a multiple reassortant, carrying gene segments from several origins. The 2009 pandemic $\mathrm{H} 1 \mathrm{~N} 1$ virus displaced the seasonal $\mathrm{H} 1 \mathrm{~N} 1$ virus but not the seasonal $\mathrm{H} 3 \mathrm{~N} 2$ virus. $\mathrm{H} 1 \mathrm{~N} 1$ and $\mathrm{H} 3 \mathrm{~N} 2$ viruses currently co-circulate, along with two lineages of influenza B virus.

Antigenic shift

Where two antigenically distinct influenza viruses co-infect a cell and reassort their genome segments, generating a novel virus with the surface antigens of one and the internal genes of the other, whereby the novel virus is of a different subtype than the virus that previously circulated in that specific host.

\section{Antigenic drift}

The continual evolution of the antigenic sites present on

surface antigens of influenza virus, driven by the need to evade the host antibody response. of HA from AIVs, the a2-3-linked SA is in a thin and straight trans conformation whereas the wider RBS of human-adapted HA accommodates the more bulky cis conformation adopted by a2-6-linked SA (reviewed in REFS $^{15,16}$ ). These alternative conformational states of the sialylated glycan have also been referred to as cone-like and umbrella-like ${ }^{17}$.

A small number of well-characterized amino acid substitutions in the AIV HA RBS can change sialylated glycan specificity, best exemplified by amino acid changes in viruses that have caused human pandemics in the 20th century, E190D/G225D in H1 HA and Q226L/G228S in H2 and H3 HAs ${ }^{18}$ (TABLE 1). The receptor binding specificity as determined by these amino acids is a key determinant of the transmissibility of virus between ferrets ${ }^{19-21}$. The ease with which these mutations can be acquired and tolerated without fitness cost to the adapting virus is a primary determinant of the likelihood that a novel AIV subtype can emerge in a transmissible form to cause a human pandemic.

Several studies have addressed whether AIVs of different subtypes that have not yet circulated in humans already have or can acquire affinity for a2-6 SA. For example, the HA of recently emerged H7N9 AIVs that have infected more than 1,625 people and resulted in 622 deaths (see Related links; FAO.org H7N9 situation update) can bind to a2-6 SA to some extent, and this might partially explain the frequent zoonoses into exposed individuals ${ }^{22}$. On the other hand, H5N1 viruses that have also infected humans retain preference 


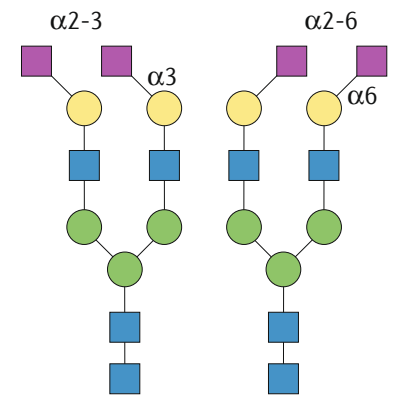

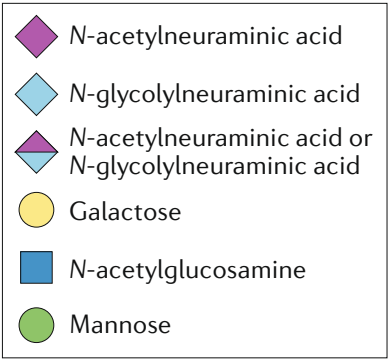

b

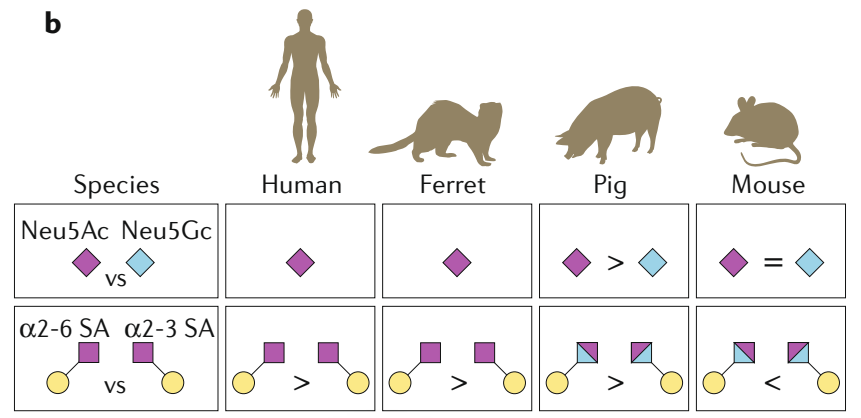

c

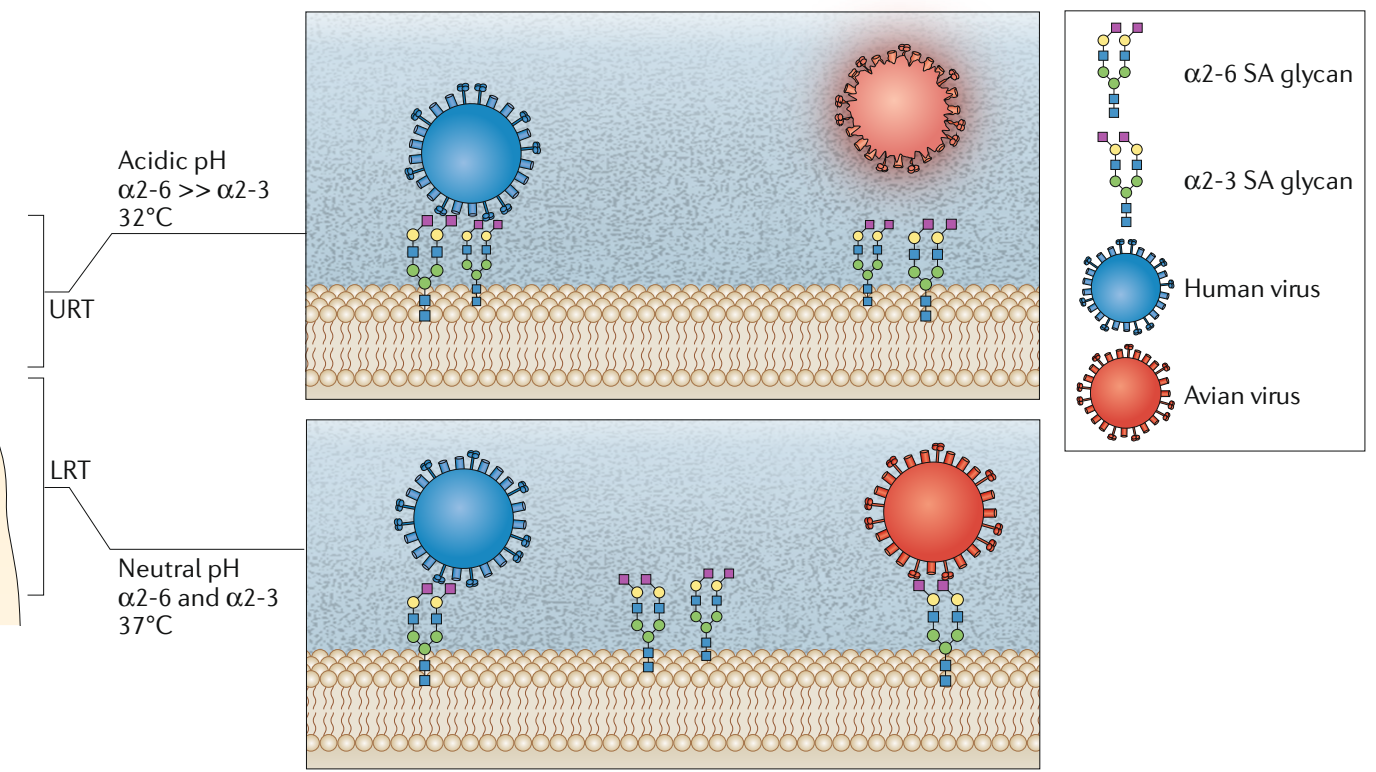

Fig. 2 | Host sialic acid presentation and physiology drives haemagglutinin adaptation. a | Example structure of a complex sialylated glycan, with either a2-3-linked or a2-6-linked sialic acid (SA). b | Species presentation of SA receptors in the respiratory tract. Human, ferret and pig respiratory tracts express a greater abundance of $a 2-6 \mathrm{SA}$ than the mouse respiratory tracts, which have equal amounts of $22-3 \mathrm{SA}$. Human and ferret respiratory tracts present $\mathrm{N}$-acetylneuraminic acid (Neu5Ac)-type SA, whereas pig and mouse respiratory tracts also present N-glycolylneuraminic acid (Neu5Gc) SA. c|SA differences and $\mathrm{pH}$ gradients exist along the human respiratory tract. $\alpha 2-6$ SA receptors predominate in the upper respiratory tract (URT), to which human influenza A virus (blue) but not avian influenza virus (AIV) (red) can bind. Both a2-3 and $\alpha 2-6$ SA receptors are present in the lower respiratory tract (LRT). The $\mathrm{pH}$ in the URT is mildly acidic and gradually increases from the URT to the LRT (reviewed in REF. ${ }^{148}$ ). The haemagglutinins (HAs) of human influenza A viruses are more $\mathrm{pH}$ stable ( $\mathrm{pH}$ 5.0-5.4) whereas the HAs of some AIVs are less $\mathrm{pH}$ stable (up to $\mathrm{pH}$ 6.1) and may be inactivated in the human URT (reviewed in REF. ${ }^{148}$ ). The temperature is lower in the URT than in the LRT ${ }^{173}$. Human influenza A virus polymerases are more active than AIV polymerases at lower temperatures ${ }^{102,174}$.

\section{Lectins}

Proteins that recognize and bind to specific carbohydrates.

Apical surface

The apical surface of a polarized epithelial cell faces the lumen or external environment. for a2-3 SA, and mutations at amino acids 226 and 228 that switch SA specificity were among those required for airborne transmissibility ${ }^{23,24}$.

The differences in the SA linkages on glycans that adorn cells can be visualized using specific plant lectins. In human respiratory tract tissue, lectin staining revealed that the majority of SAs at the apical surface of cells are a2-6 linked ${ }^{25}$. This pattern is most marked in upper respiratory tract (URT) tissues; for example, the nasopharyngeal surface of the soft palate, which may be the primary site for entry of transmitted viruses ${ }^{26}$ (FIC. 2C). Glycomic characterization confirmed the presence of both a2-3 SA and $\alpha 2-6 \mathrm{SA}$ in the human lung and bronchus ${ }^{27}$ (FIG. 2c). a2-6 SAs are also prevalent in the respiratory tract of pigs and ferrets ${ }^{28,29}$. By contrast, non-human primates and mice show higher expression of $\alpha 2-3 \mathrm{SA}$, explaining why they are not good models for human-adapted influenza viruses ${ }^{30,31}$. Using labelled virus or expressed HA proteins as probes corroborates these patterns in which viruses with HAs known to prefer $\alpha 2-3$ SA bind to cells in human lung sections, whereas seasonal influenza virus HAs bind to the apical surface of URT human tissue $^{32,33}$. In birds, there is great variation in the type and distribution of $\mathrm{SA}^{34}$. Duck intestine and trachea exclusively display a2-3 SA, whereas chickens show evidence of both linkages in the respiratory $\operatorname{tract}^{35}$. Higher expression of a2-6 SA on quail cells has led to suggestions that quail and other terrestrial birds might be intermediates in the adaptation of avian viruses to mammals ${ }^{36,37}$.

The view that the linkage of the terminal SA residue is fundamental to HA species specificity still holds today, but it is not yet clear how avidly HA must bind to a2-6 SA to support airborne transmission between humans. Whether concomitant loss of a2-3 SA binding is also 
Table 1 | Major viral determinants of host range for influenza A virus pandemics

\begin{tabular}{llll}
\hline Amino acid & Avian & Human & Refs \\
\hline Gene segment PB2 & & & \\
\hline 271 & T & A & 60,125 \\
\hline 590 & G & S & $98-100$ \\
\hline 591 & Q & R & $98-100$ \\
\hline 627 & E & K & 96,97 \\
\hline 701 & D & N & $83-86$ \\
\hline Gene segment HA $(H 1)$ & & & \\
\hline 190 & E & D & 13,18 \\
\hline 225 & G & D & 13,18 \\
\hline Gene segment HA $(H 2$ and H3) & & & 13,18 \\
\hline 226 & G & S & 13,18 \\
\hline 228 & Q & L & 13,18 \\
\hline 192 & R & G & 13,18 \\
\hline 226 & Q & L & \\
\hline
\end{tabular}

HA, haemagglutinin; PB2, polymerase basic protein 2 .

required is also not established. However, it is becoming clear that this simple model will have to be extended to include other structural features of sialylated glycans.

Other glycan structural features contributing to host range. It is not only the terminal SA linkage that affects the interplay between influenza virus HA and its receptor. Additional structural features of glycans, such as modifications with other sugar moieties (such as fucose and $\mathrm{N}$-acetylgalactosamine) or other functional groups (such as sulfation), and the length of the SA-presenting glycan chain all affect the interaction. Such detailed glycan structural features are not resolvable by lectin staining and require mass-spectrometry-based glycomic analysis. To date, glycomic analysis has been carried out on human, ferret, pig and mouse respiratory tissue, all of which express a complex set of protein $N$-linked and $O$-linked glycans as well as glycolipids ${ }^{27,29,38-40}$. As expected from the knowledge of CMAH genetics, humans and ferrets express only Neu5Ac SA, whereas pig and mouse express both Neu5Ac and Neu5Gc SA. All the tissues analysed express both $\alpha 2-3$ SA and $\alpha 2-6$ $\mathrm{SA}$, with humans expressing the highest proportion of a2-6 SA and the mice expressing the highest proportion of a2-3 SA. However, different glycan structural features can be observed in individual species. For example, uniquely in ferret respiratory tract tissue, the Sda epitope blocks access of HA to a2-3 SAs, potentially restricting their ability to support the transmission of $\mathrm{AIVs}^{41}$. This inhibitory effect on AIV was recapitulated in human and canine cell lines engineered to overexpress the B4GALNT2 gene, which generates the Sda epitope ${ }^{42}$. Human respiratory tract tissue is rich in large complex $\mathrm{N}$-glycans with sialylated poly- $\mathrm{N}$-acetyl-lactosamine (LacNAc) chains. This topology may allow bivalent interactions with two of the three monomers of the HA trimer ${ }^{41}$ and substantially increase the avidity of the HA-SA interaction, supporting airborne transmission.

It is becoming clear that detailed characterization of the sialylated glycome from different anatomical sites in different host species, especially birds, is required. Only when we combine knowledge of the relevant glycans identified from primary tissues (for example, from shotgun glycan array experiments ${ }^{40}$ ) with crystallographic structural data for different subtypes of HA will we gain a detailed molecular understanding of this important interaction that initiates infection.

Viral neuraminidase displays species-specific adaptation. Detailed glycomic analyses of tissues from avian species are currently lacking. However, it is tempting to speculate that a difference in glycan topology might drive selection of viruses with a short-stalk neuraminidase (NA), often observed in poultry ${ }^{43}$. NA is a membrane-bound viral sialidase found on the surface of infected cells and virions. NA cleaves SA from the cell surface during the final stages of the replication cycle, enabling the release of progeny virions (FIG. 3). One could speculate that if poultry SA receptors are at the termini of short glycan chains, then viral NA should extend only a short distance from the cell surface to sterically match its active site with its substrate. Viruses bearing short-stalk NA are compromised for airborne transmission between ferrets, possibly owing to an inability of short-stalk NA to cleave decoy receptors present in airway mucus or on HA on virions, where ineffective cleavage would result in virus clumping ${ }^{44}$. Indeed, a functional balance between HA affinity for SA and NA enzymatic activity for relevant substrates is undoubtedly essential to support airborne transmission between humans $s^{45-47}$. Some NA proteins harbour a second SA binding site in addition to the enzymatic site - a haemadsorption $(\mathrm{Hd})$ site $^{48}$. Recent analysis of the N9 NA protein of H7N9 viruses suggested that its $\mathrm{Hd}$ site contributes to receptor binding ${ }^{49}$, but the relevance of this to the large number of human cases of infection with this AIV is not yet established. Other data suggest that the Hd site of NA is lost during human adaptation $^{48}$.

Undoubtedly, differences in glycan composition and SA specificity in different host species drive co-evolution of both HA and NA. Viruses that infect and transmit between humans preferentially bind long glycans that are 22-6 sialylated, allowing efficient interactions with target tissues in the human respiratory tract. HA of AIVs must acquire RBS mutations that enable new binding specificity. We should consider that HA and NA are also glycosylated, and this could have consequences for immune recognition, enzyme function and receptor affinity. Changes in the extent of HA glycosylation selected for in one host may affect the virus's potential to cross-infect a different species ${ }^{23,24}$.

\section{Virus genome delivery to the nucleus}

After the virus has attached to the cell, it is endocytosed and the decrease in $\mathrm{pH}$ within the endosome triggers an irreversible conformational change in HA that initiates a series of events that lead to the release of viral ribonucleoproteins (vRNPs) into the cytoplasm (FIG. 3c). The virus co-opts host factors to support uncoating, genome release and vRNP transport to the nucleus ${ }^{50}$. vRNPs in transit from the cytoplasm to the nucleus can be targeted 


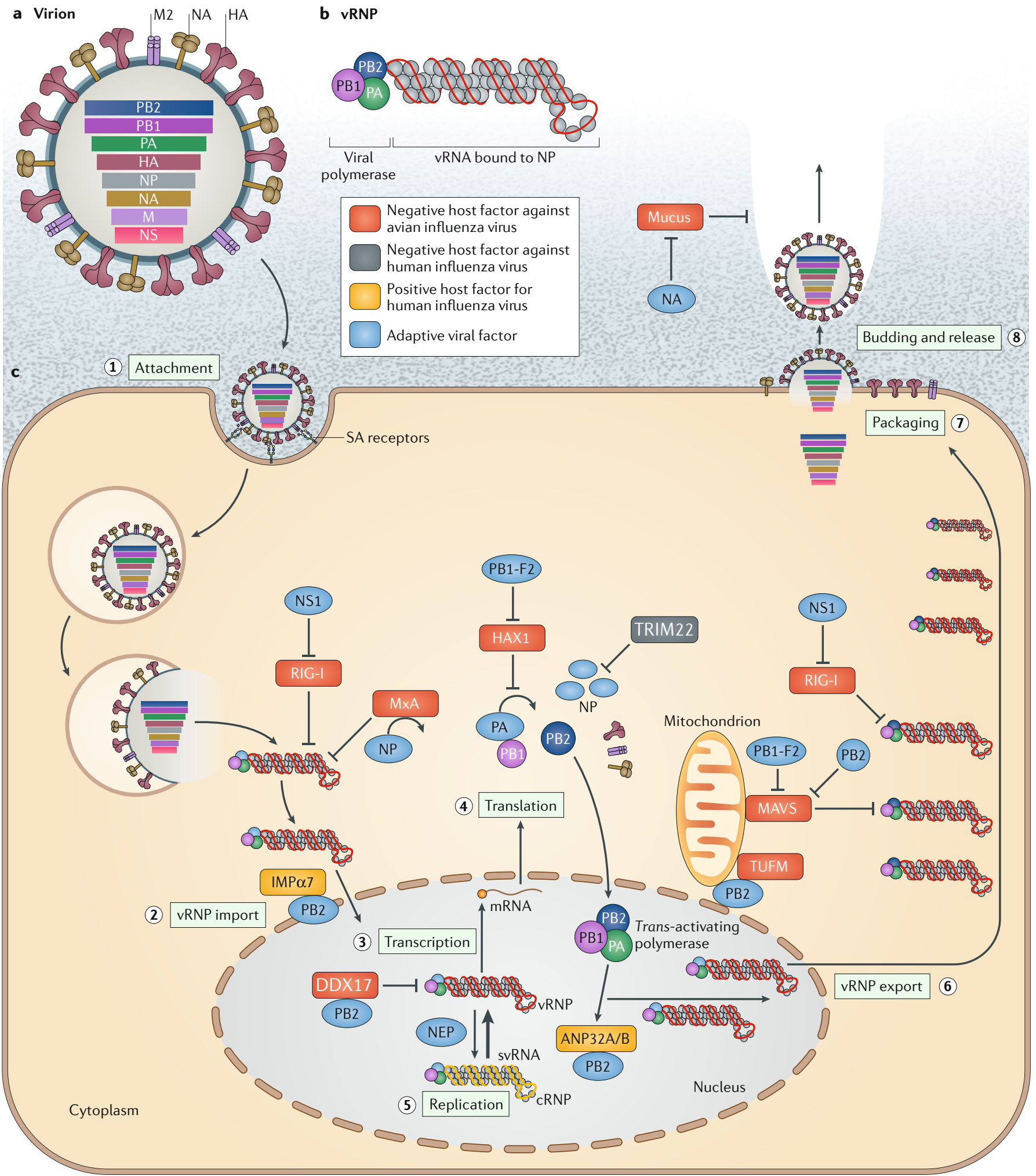

by host restriction factors ${ }^{51}$, and unadapted AIVs are particularly susceptible to this block in mammalian cells.

Sensing or blockade by the innate immune system during cytoplasmic transit. Retinoic acid-inducible gene I protein (RIG-I also known as DDX58) is a cytoplasmic pattern recognition receptor best known for its role in sensing RNA products of virus replication. RNA binding by RIG-I induces a conformational change that exposes the caspase recruitment domain (CARD), allowing interaction with mitochondrial antiviral signalling protein (MAVS), leading to the expression of type I and type III interferons ${ }^{52}$. These interferons are secreted from the cell and bind to cell surface receptors on the infected and neighbouring cells, inducing a signalling cascade that triggers expression of hundreds 
4 Fig. 3 | Virus and host-specific determinants of influenza virus replication. In human cells, avian influenza viruses (AIVs) may encounter negative host factors (red) that human-adapted viruses overcome by viral gene adaptation (blue). AIVs may be unable to co-opt positive human host factors (gold) and human-adapted viruses may develop susceptibility to restriction factors (dark grey). a | Influenza A viruses are enveloped viruses containing eight single-stranded negative-sense RNA gene segments that each encode one or more proteins. $\mathbf{b}$ | Each RNA segment (red line) surrounds an oligomeric nucleoprotein (NP) structure (light grey), and the viral promoters are bound by the heterotrimeric polymerase complex containing polymerase basic proteins 1 (PB1) and 2 (PB2) and polymerase acidic protein (PA); the entire structure is known as the viral ribonucleoprotein (vRNP) complex. The eight segments encode ten essential viral proteins: PB1, PB2, PA and NP for RNA replication; non-structural protein 1 (NS1) to evade the host innate response; matrix protein $\mathrm{M} 1$ as the main structural matrix of the virus particle; nuclear export protein (NEP) for nuclear export of vRNPs; and haemagglutinin $(\mathrm{HA})$, matrix protein $\mathrm{M} 2$ and neuraminidase (NA), which are embedded in the virion envelope for virus attachment, uncoating and onward spread, respectively. In addition, several accessory proteins such as protein PB1-F2, PB1-N40, PA-X, PB2-S1, matrix protein M42 and NS3 may be encoded by some but not all virus strains. $\mathbf{C}$ /Virus infection begins with cell attachment mediated by binding of $\mathrm{HA}$ to sialic acid (SA) receptors on the cell surface (step 1). Entry of the virion is by endocytosis, and the decrease in $\mathrm{pH}$ within the endosome triggers an irreversible conformational change in HA that exposes the hydrophobic fusion peptide and stimulates the fusion of the viral envelope with the endosomal membrane ${ }^{154}$. At the same time, the passage of protons and potassium ions through the small ion channel $\mathrm{M} 2$ acidifies the virion interior and mediates dissociation of M1 from vRNPs, releasing the vRNPs into the cell cytoplasm ${ }^{175,176}$. Interferon-regulated resistance GTP-binding protein MxA and retinoic acid-inducible gene I protein (RIG-I) restrict AIV vRNPs in the cytoplasm. Mutations in human-adapted NP evade MxA binding. Human-adapted vRNPs with PB2 627K are less vulnerable to RIG-I detection. vRNPs are imported into the nucleus (step 2). Humanizing mutations in PB2 enable importin- $\alpha 7$ (IMPa7; also known as KPNA6) use and enhance vRNP nuclear import. Primary transcription occurs in cis and in conjunction with the cellular RNA polymerase II (step $3)^{177}$. Viral mRNAs are exported to the cytoplasm for translation by the cellular machinery (step 4). Human-adapted NP is susceptible to degradation by tripartite motif-containing protein 22 (TRIM22). AIV PA is susceptible to inhibition by HS1-associating protein X1 (HAX1), but this can be counteracted by protein PB1-F2. The newly synthesized polymerase and NP proteins are imported into the nucleus to carry out replication and further (secondary) transcription (step 5). Acidic leucine-rich nuclear phosphoprotein 32 family member A (ANP32A) or B (ANP32B) and small viral RNAs (svRNAs) promote viral RNA (vRNA) production from complementary RNA (cRNA) (yellow line). AIV polymerase cannot utilize human ANP32A or ANP32B and must gain human-adapting mutations such as PB2 627K. NEP can also overcome the AIV polymerase restriction. DEAD box protein 17 (DDX17) restricts AIV polymerase PB2 627E and promotes activity of human-adapted polymerase containing PB2 627K. Newly formed vRNPs are exported to the cytoplasm by $\mathrm{M} 1$ and the NEP ${ }^{178}$ (step 6). vRNPs and other viral products are detected by multiple cell sensors. Mitochondrial Tu elongation factor (TUFM) binds to AIV PB2 containing 627E, increasing autophagy and decreasing virus production. Human-adapted PB2 and protein PB1-F2 localize to mitochondria and prevent detection by mitochondrial antiviral signalling protein (MAVS). NS1 binds to RIG-I to prevent activation. vRNPs transported to the cell surface for packaging are assembled with the structural proteins (HA, NA, M1 and M2) (step 7). Progeny virions are formed by budding from host cell plasma membrane, and release is mediated by scission of the membrane by M2 (step 8). Onward spread is facilitated by the removal of SA from the cell surface, from viral particles and from mucus by $\mathrm{NA}^{179}$. Short-stalk avian NAs are unable to escape human respiratory tract mucus; human-adapted viruses with long-stalk NAs can overcome this restriction.

Interferon-stimulated genes (ISGs). A set of genes for which transcription is stimulated following engagement of the soluble cytokine interferon with its receptor. ISGs exert antiviral effects within the cell. mammalian-adapted viruses. The interaction between the viral nucleoprotein (NP) and viral polymerase in vRNPs appears to be less stable when glutamic acid is present at position $627\left(\mathrm{REFS}^{55,56}\right)$. This less-stable interaction may allow RIG-I access to viral RNA in the AIV vRNP. Interestingly, chickens lack a functional RIG-I homologue; thus, incoming vRNPs are not blocked in this way in chicken cells ${ }^{57}$. However, RIG-I is expressed and active in ducks, geese and pigeons, which are natural hosts of AIVs that retain PB2 627E ${ }^{58}$. Indeed, the PB2 mutation E627K does not confer any replicative advantage to influenza virus in ducks ${ }^{59}$, suggesting that RIG-I susceptibility is not a primary driver of the host-adapting PB2 E627K mutation, and the decreased interaction between NP and PB2 627E in human cells may simply be a reflection of a less active viral polymerase ${ }^{60}$.

A second, interferon-induced host factor that may restrict influenza virus during vRNP transport to the nucleus is interferon-regulated resistance GTP-binding protein MxA (also known as Mx1). Mouse Mx1 protein (the mouse orthologue of $\mathrm{MxA}$ ) is a powerful nuclear ISG that inhibits influenza virus transcription in the nucleus, and its expression in wild mice may explain their natural resistance to influenza viruses ${ }^{61}$. Human $\mathrm{MxA}$ is a cytoplasmic protein that can inhibit nuclear import of $\mathrm{vRNPs}^{62,63}$, although additional proteins may be required to support MxA anti-influenza virus activity $^{64}$ (FIG. 3C). The Mx proteins of chickens do not restrict influenza virus replication ${ }^{65}$. The vRNPs of AIVs were found to be more sensitive to human MxA inhibition than those from human-adapted influenza virus, and this mapped to a patch of amino acids on $\mathrm{NP}^{66-68}$ (FIG. 3C). Indeed, transgenic mice that possess the human $M x A$ locus were resistant to highly pathogenic $\mathrm{H} 5$ or H7 AIV infection, unless the virus possessed mutations in NP that evaded MxA inhibition ${ }^{69}$. Interestingly, Mx-evading mutations in NP decrease virus fitness because they affect the efficiency of nuclear import of $\mathrm{vRNP}^{70}$. Some AIVs, such as the H7N9 influenza viruses that emerged in China in 2013, already possess some MxA-evading NP mutations, and this, in addition to their ability to bind a 2-6 SA receptors to some extent, may potentiate their ability to cross into humans ${ }^{71}$.

Several other restriction factors have been characterized that inhibit the early stages of influenza virus infection: interferon-induced transmembrane protein 3 (IFITM3) hinders the fusion of the viral envelope with the endosomal membrane, and this inhibits the release of vRNPs from endosomes ${ }^{72}$. Other human restriction factors including DEAD box protein 21 (DDX21) $\left(\mathrm{REF}^{73}\right)$, Moloney leukaemia virus 10 protein (MOV10) $\left(\mathrm{REF}^{74}{ }^{74}\right.$, guanylate-binding protein 3 (GBP3) $\left(\mathrm{REF}^{75}\right), \mathrm{HS} 1$ associating protein X1 (HAX1) (REF. ${ }^{76}$ ), TRIM22 (REF. ${ }^{77}$ ) and TRIM25 (REF. ${ }^{53}$ ) inhibit the viral polymerase, some by directly binding vRNP components. For most of these factors, there is no evidence as yet that their inhibitory effect is more potent against AIVs. Instead, for IFITM3 and TRIM22, the opposite might apply as human viruses are more vulnerable to IFITM3 (REF. ${ }^{78}$ ) than AIV and appear to have accumulated mutations in NP that render them more susceptible to TRIM22. The mutations in NP allow human influenza viruses to escape T cells that 
target $\mathrm{NP}^{79}$. However, recent data illustrated that HAX1 impeded nuclear import of an avian polymerase acidic protein (PA) but did not restrict human-adapted PA proteins. Interestingly, for some viruses, including zoonotic H5N1 AIVs, protein PB1-F2 (FIG. 3c) was able to counter the restriction mediated by HAX1 (REF. ${ }^{80}$ ).

Nuclear import. Members of the importin- $\alpha$ family actively import vRNPs into the nucleus through the nuclear pore ${ }^{81}$ (FIG. 3C). Following primary transcription and translation of viral proteins, importin- $\alpha$ proteins also mediate nuclear import of the newly synthesized $\mathrm{NP}$ and $\mathrm{PB} 2$ proteins required to sustain virus replication. Importin- $\alpha$ proteins have been strongly implicated in influenza A host-specific adaptation ${ }^{82,83}$. Mutations that arose in PB2 and NP during mammalian adaptation of an AIV (PB2 D701N and NP N319K) enhanced vRNP binding to human importin- $\alpha 1$, which increased the efficiency of vRNP nuclear import in human cells ${ }^{84-86}$. Residue 701 is located adjacent to a PB2 nuclear localization signal (NLS) and conformational changes induced by the D701N mutation likely enhance access to importin- $\alpha^{85-87}$.

In addition to their role in nuclear import, importins may have a role in supporting viral replication within the nucleus. When the authentic NLS in the carboxy-terminal region of the $\mathrm{PB} 2$ protein was replaced with an exogenous NLS, polymerase activity was abrogated $^{88}$. This finding, along with the observation that importin- $\alpha$ was retained in the nucleus in cells infected by mammalian-adapted viruses possessing PB2 $701 \mathrm{~N}$ or NP 319K, suggests that importin- $\alpha$ may remain associated with the viral polymerase within the nucleus and directly support its function there or recruit other host factors ${ }^{84}$. From a host range perspective, specific members of the importin- $a$ family may be key in this role. In human cells, small interfering RNA (siRNA)mediated knockdown of importin- $\alpha 1$ or importin- $\alpha 7$ reduced the polymerase activity of a human-adapted PB2-627K-containing polymerase but had no effect on a polymerase with PB2 $627 \mathrm{E}^{89,90}$. The greater abundance of importin- $\alpha 7$ mRNA than other importin- $\alpha$ isoforms in human nasal epithelia may drive adaptation of human viruses towards this specific importin- $\alpha$ protein ${ }^{91}$.

Although the loss of specific importin- $\alpha$ isoforms undoubtedly inhibits the replication of mammalianadapted viruses, as indicated by decreased replication in cells and in knockout mice, the mechanism by which importin- $\alpha$ proteins support polymerase activity in the nucleus is unknown ${ }^{89,90}$. Indeed, it is important to note that the importin- $\alpha$ proteins facilitate entry of many cellular proteins into the nucleus. Whether they support influenza virus polymerase through a direct interaction, by recruiting an additional cofactor or by delivering a host protein that is required for polymerase activity into the nucleus, is not clear.

\section{Host specificity of the viral polymerase}

Once the vRNPs have entered the nucleus, the genome is first transcribed and then replicated. Replication depends on the delivery of newly synthesized polymerase to stabilize the complementary ribonucleoprotein (cRNP) and to transactivate replication of new genomes (reviewed in $\mathrm{REFS}^{92,93}$ ) (FIG. 3C). AIV polymerases perform genome replication (rather than transcription) poorly in mammalian cells ${ }^{94,95}$. Multiple host-adaptive mutations in viral polymerase subunits have been described that partially overcome this major host range barrier (reviewed in REF. ${ }^{95}$ ). The best known is the aforementioned PB2 E627K mutation ${ }^{96}$. The $627 \mathrm{~K}$ mutation was present in the PB2 of the $1918 \mathrm{H} 1 \mathrm{~N} 1$ pandemic virus and was retained during the reassortment events that led to the $1957 \mathrm{H} 2 \mathrm{~N} 2$ and 1968 H3N2 pandemic viruses; thus, all human circulating viruses of the 20th century possessed this signature ${ }^{97}$ (BOX 1). By contrast, the 2009 pandemic virus had PB2 $627 \mathrm{E}$, but its ability to support efficient replication in human and pig cells is explained by alternative mutations in PB2 (G590S and Q591R), located adjacent to residue 627 in the heterotrimeric polymerase ${ }^{98-100}$. The PB2 627 domain is a solvent-exposed region of the polymerase, and both $627 \mathrm{~K}$ and $591 \mathrm{R}$ adaptive mutations increase the degree of positive charge in this region, suggesting that they affect interactions with a host or viral factor ${ }^{101}$. The AIV polymerase is sensitive to cold, and its low activity in the human URT might be partially explained by this ${ }^{102}$ (FIG. 2c). However, the fact that purified avian-derived and mammalian-derived influenza polymerases have similar levels of activity in in vitro assays suggests that the block to AIV polymerase is explained by incompatibility with specific host factors ${ }^{103}$.

The activity of influenza virus polymerases can be measured in situ by reconstituting vRNPs in cells together with a minigenome reporter that expresses a single-stranded viral-like RNA flanked by viral non-coding regions that form the viral promoter ${ }^{104}$. Restriction of AIV polymerase activity measured by this minigenome reporter assay in mammalian cells could be overcome by fusion with increasing numbers of avian cells to form heterokaryons that contained cellular factors from both species. This result implied that the poor activity of AIV polymerase in the human nucleus was rescued by introducing a supportive avian host factor that is either lacking or different in mammals ${ }^{105}$. This factor was identified to be chicken acidic leucine-rich nuclear phosphoprotein 32 family member A (ANP32A) ${ }^{106}$ (FIG. 3C). ANP32A contains a multiple leucine-rich repeat (LRR) domain and a low complexity acidic (LCAR) domain. Both of these domains have the capacity to bind to various cellular proteins, which accounts for the plethora of functions attributed to ANP32 family members, including roles in development, regulation of apoptosis, mRNA export and chromatin remodelling (reviewed in REF. ${ }^{107}$ ).

Exogenous expression of chicken ANP32A increased the activity of AIV polymerase possessing PB2 627E in human cells from almost undetectable levels to an activity similar to that seen for the polymerase containing the PB2 $627 \mathrm{~K}$ mutation ${ }^{106}$. The human homologues (ANP32A and ANP32B) support replication of human-adapted, but not of AIV, polymerase; knockdown of these proteins in human cells resulted in decreased viral polymerase activity in a minigenome reporter assay and reduced virus growth ${ }^{106,108,109}$. Similarly, knockdown of chicken ANP32A abrogated replication in chicken cells. An important difference between avian and 
SUMO interacting motif

(SIM). The amino acid sequence found on cellular proteins recognized by small ubiquitin-like modifier (SUMO) proteins.

Ratites

Birds including the ostrich, emu, rhea and kiwi belonging to the Palaeognathae. mammalian ANP32A proteins explains the host range barrier for AIV polymerase activity. Avian ANP32A orthologues encode an additional 33 amino acids between the two domains of the protein that are crucial for their ability to support AIV polymerase activity and act to enhance their interaction with the viral polymerase ${ }^{106,110}$. A SUMO interacting motif (SIM) located in the first 4 amino acids of the 33-amino-acid insert may confer the increased binding to the polymerase complex ${ }^{110}$. The latter 27 amino acids in the avian ANP32A gene result from a duplication event during evolution of flighted birds and are lacking in ratites such as the ostrich, emu and rhea. A splice variant of chicken ANP32A that lacks the SIM motif can also support viral polymerase activity, although with less potency than with all 33 amino acids ${ }^{110,111}$. In accordance with the concept that PB2 E627K is an adaptation towards utilizing short ANP32 homologues, viral replication in ostriches selected for this humanizing mutation $^{112}$. Both avian and mammalian ANP32B orthologues lack the 33-amino-acid duplication, yet artificial insertion of this sequence between the LRR and LCAR domains of human ANP32B enabled AIV polymerase activity when co-expressed in human cells ${ }^{106}$.

The exact role of ANP32 proteins in virus replication remains to be elucidated. Co-immunoprecipitation experiments from infected and transfected cells revealed an interaction between ANP32A or ANP32B and the viral polymerase trimer ${ }^{109-111,113}$. In vitro binding assays with purified proteins showed that this interaction maps to the 627 domain of PB2 (REF. ${ }^{111}$ ). Interestingly, however, this binding is not dependent on the nature of the amino acid at position 627 . The interaction between chicken ANP32A and the viral polymerase increases upon binding to viral genomic $\mathrm{RNA}^{111}$. As the viral polymerase can adopt multiple conformations depending on the presence or absence of specific RNAs, it is likely that ANP32A prefers binding to specific conformations $s^{99,114-116}$. Mutations at positions 3 and 8 of the $3^{\prime}$ viral RNA (vRNA) promoter region can also increase polymerase activity of a restricted polymerase $\mathrm{e}^{117-119}$. Chicken ANP32A could not further potentiate replication of RNAs with these mutations, suggesting that ANP32A and mutations in the RNA overcome the same block ${ }^{111}$. ANP32A may act by delivering the trans-acting or trans-activating polymerase to cRNPs, or it might be required for loading the viral polymerase complex onto nascent vRNPs. Of note, cRNPs can be stabilized by an avian viral polymerase in human cells, but they do not synthesize vRNA ${ }^{94,120}$. Indeed, the in vitro replication of RNA from a short complementary RNA (cRNA) template is promoted by human ANP32A and ANP32B ${ }^{109}$. Together, this may suggest that chicken ANP32A enables the avian viral polymerase to synthesize vRNA from cRNPs in human cells, although further experimental evidence is still required to confirm this.

In other experiments involving fusion between human and avian cells, AIV polymerase activity was decreased in the heterokaryons, implying the existence of a human-specific restriction factor ${ }^{56}$. DDX17 was revealed as a candidate for this factor, as siRNA knockdown of DDX17 resulted in increased polymerase activity of AIV polymerase in human cells. By contrast,
DDX17 was found to have a role in supporting the activity of a polymerase possessing the PB2 627K signature as siRNA decreased polymerase activity. Furthermore, avian DDX17 was required for AIV polymerase activity in avian cells. This finding suggests incompatibility between the unadapted AIV polymerase and human DDX17 (REF. ${ }^{121}$ ) (FIG. 3c). DDX17 bound more strongly to AIV polymerase than human influenza virus polymerase, suggesting that strong binding inhibits viral polymerase activity. However, overexpression of DDX17 in human cells did not inhibit AIV polymerase activity. Thus, the role of DDX17 in host range restriction of the influenza virus polymerase is not clear.

It remains likely that additional mammalian host factors affect AIV polymerase activity. Many other mutations in polymerase proteins enable AIV to replicate in human cells, but the corresponding host partners are not yet identified $^{95,122}$. For example, T552S in PA increases the replication of an AIV polymerase in mammalian cells but has no effect in avian cells ${ }^{123}$, and mutations in PB2 that map distally from the 627 domain, such as E158G or T271 $\mathrm{A}^{124,125}$, may enhance polymerase activity in human cells through a different mechanism than E $627 \mathrm{~K}^{60}$. It is not always clear whether these mutations overcome the host range barrier in a species-dependent manner or whether they increase the overall activity of polymerase regardless of host species and thus simply enable restriction by host factors to be overcome ${ }^{60}$.

Mutations in the nuclear export protein (NEP) also enable AIV to overcome the replication block in mammalian cells ${ }^{94}$ (FIG. 3c). This finding is particularly apparent in minigenome reporter assays; co-expression of NEP in human cells with mammalian-adapted polymerase (PB2 627K) promoted the accumulation of cRNA and supressed the accumulation of viral mRNA, resulting in a decrease in minigenome expression ${ }^{126}$. Conversely, co-expression of NEP enhanced AIV polymerase activity in human cells ${ }^{94}$. There does not appear to be a host factor involved in this effect. An interaction between NEP and the PB1 and PB2 polymerase subunits was mapped to the carboxy-terminal domain of $\mathrm{NEP}^{94,126,127}$, the exposure of which depends on the sequence at the amino terminus. For example, the M16I that is present in the pandemic H1N1 2009 virus and a human isolate of H5N1 AIV promotes the exposure of the carboxy-terminal domain ${ }^{127}$. It is suggested that adaptation to replication in the cooler temperatures of the human URT requires an NEP that can adopt the polymerase-enhancing conformation more readily than that of a typical AIV, which replicates in the warmer tissues of birds ${ }^{94}$. It is not yet clear how exposure of the carboxy terminus of NEP enhances polymerase activity, but the terminal three highly conserved amino acids are essential for this function ${ }^{127}$. Other studies have identified short viral RNAs (svRNAs) that promote the synthesis of vRNA from cRNA templates in a segment-specific manner and the generation of which depends on NEP expres$\operatorname{sion}^{127,128}$. svRNAs were detected in both mammalian and avian cells ${ }^{129}$. Whether the ability of NEP to overcome the host range block is linked to an increase in svRNA accumulation, a difference in its ability to export vRNPs from the nucleus at later time points or an enhanced ability to 
induce AIV polymerase to switch from transcription to replication is not yet clear ${ }^{130}$.

To summarize, influenza virus genome replication represents one of the major host range barriers. Differences in host proteins that promote or restrict replication, as well as differences in the temperature at which the polymerase enzyme activity is optimal and properly regulated, contribute to this block. Mutations in all three viral polymerase genes, as well as in NP and NEP, can enhance AIV polymerase activity in mammalian cells, although the mutation E627K in PB2 is the most potent enhancer. Whether the route to viral polymerase adaptation affects disease severity is an interesting and unanswered question.

\section{Evasion of the host response}

During infection, an antiviral response is triggered and ISGs are produced to limit infection. The exact nature of the influenza virus pathogen-associated molecular patterns (PAMPs) is not agreed upon. An RIG-I response requires vRNAs to be produced by an actively replicating polymerase, but whether these are full-length products or shorter RNAs produced by polymerase errors is not clear ${ }^{52,131}$. Evidence also shows that cellular-derived RNAs can escape the nucleus and trigger RIG-I in the cytoplasm during influenza virus replication ${ }^{132}$. Although all influenza viruses counter the cellular innate immune response to an extent by encoding interferon antagonists or masking PAMPs, the outcome of infection is a balance determined by the levels of stimulatory viral RNAs, the expression and potency of virally encoded interferon antagonists, the capacity of the host cell to mount and respond to an interferon response and the susceptibility of the virus to the ISGs. As previously mentioned, AIVs are particularly susceptible to some human ISGs, including RIG-I and $\mathrm{MxA}^{54,67}$. In human cells lacking an interferon response, AIVs that were otherwise restricted could replicate to some extent ${ }^{133}$.

Influenza A virus encodes a number of viral proteins that counter the cellular innate immune response. The major viral interferon antagonist is non-structural protein 1 (NS1), encoded on RNA segment 8, that also encodes NEP by alternative splicing. NS1 limits interferon induction in at least two ways: by counteracting RIG-I activation and by binding to CPSF30 (Cleavage and polyadenylation specificity factor $30 \mathrm{kDa}$ subunit; also known as CPSF4) and preventing the processing of newly synthesized mRNAs, including interferon- $\beta$ mRNAs. Despite the many different host factors that NS1 can bind, mutations in NS1 are not readily linked to host range. The NS1 of most AIVs can bind human CPSF30 and is an efficient interferon antagonist in human cells, and recombinant human influenza viruses with segment 8 from AIV were not attenuated ${ }^{133,134}$. The pandemic H1N1 2009 virus cannot bind CPSF30 and yet was not restricted in human hosts ${ }^{135}$. H5N1 viruses that infected humans in 1997 also could not bind CPSF30, but viruses associated with human infections in 2003 and later could ${ }^{136}$. Human infection with both early (1997) and later (post-2003) $\mathrm{H} 5 \mathrm{~N} 1$ viruses was associated with very high viral loads and a high induction of pro-inflammatory cytokines. By contrast, adaptation of AIV in the equine host appears to have selected against CPSF30 binding and towards a more focused antagonism of the interferon signalling pathway ${ }^{137}$. It is likely that the balance between replication and interferon antagonism is askew during a host range breach and the loss or gain of CPSF30 binding may be a mechanism by which this imbalance can be addressed during adaptation to the new host.

Some variations in the RNA sequence of segment 8 may affect replication capacity in different hosts by altering the extent of mRNA splicing and thus the relative amounts of NS1 and NEP generated in the cell. Splicing of segment 8 mRNA is regulated by cis-acting RNA elements, NS1 and host factors including serine/ arginine-rich splicing factor 1 (SF2; also known as SRSF1), heterogeneous nuclear ribonucleoprotein $\mathrm{K}$ (hnRNP K) and influenza virus NS1A-binding protein $(\mathrm{NS} 1 \mathrm{BP})^{138}$. Some influenza viruses that transmitted from avian to mammalian hosts harbour an additional splice donor site that generates NS3, a variant of NS1 that harbours an internal deletion. However, no function has been assigned to this protein ${ }^{139}$. Some recent AIVs that have infected humans display a variation in the exonic splicing enhancer element in segment 8 mRNA that reduced the ratio of NEP to NS1 and increased replication in human cells ${ }^{140}$. This finding suggests that regulating the levels of NEP and NS1 during infection is important and that it may be beneficial for an AIV in a human cell to generate more NS1 early in infection to evade the host antiviral response and increase replication.

Another host response that limits influenza virus replication is autophagy. Autophagy is a complex cellular process that can be a defence against microorganisms and viruses. Indeed, influenza virus can evade autophagy through interactions between its matrix protein M2 and the microtubule-associated protein 1 light chain $3 \beta$ protein $^{141}$. Recently, autophagy was suggested to have a role in limiting AIV replication in human cells. The mitochondrial Tu elongation factor (TUFM) was found to differentially bind to avian virus-like PB2 bearing 627E, inhibiting virus replication in an autophagy-dependent manner. Polymerase activity itself was not affected by TUFM ${ }^{142}$ (FIG. 3c). However, it should be noted that this study was carried out using a human virus that was mutated to carry the avian-like motif PB2 627E whereas the PB2 protein of most AIVs does not locate to the mitochondria because they lack an amino-terminal mitochondrial targeting sequence (MTS) ${ }^{143}$. Whether TUFM inhibition is relevant for regulation of AIVs is not yet clear. Similarly, the drivers of acquisition of mitochondrial targeting of PB2 by mammalian-adapted viruses are not known, neither is the function for PB2 within the mitochondria, although studies suggest it interferes with MAVS signalling and thus helps to counter the induction of interferon ${ }^{143}$.

The viral accessory protein PB1-F2 associates with the mitochondria, disrupts MAVS signalling and promotes apoptosis $^{144,145}$. Protein PB1-F2 shows variation between viruses adapted to different species; the vast majority of AIVs retain full-length PB1-F2 expression, and its presence appears to enhance virus replication and spread between avian hosts ${ }^{146}$. In viruses adapted to swine or humans, the PB1-F2 ORF is often truncated or lost. Loss of PB1-F2 from mammalian viruses might be explained by 
compensation of its anti-interferon function by mitochondrial location of the PB2 protein of mammalian-adapted viruses $^{143}$ or because it is no longer needed to counter HAX1 inhibition of human-adapted $\mathrm{PA}^{80}$.

It seems likely that the different expression patterns of innate detection and antiviral effector molecules in different species will drive adaptation of influenza viruses when they infect a new host. This adaptation can involve changes that alter the binding partners as well as the relative expression or cellular location of viral antagonists of the cellular innate immune response.

When a virus that has been enzootic in one species is introduced to another, the initial incompatibility with the new host can cause severe disease because the virus triggers inappropriate host responses such as a cytokine storm, as is often associated with infection of partially adapted AIVs in humans ${ }^{147}$.

\section{Environmental and physiological factors}

The final stage of the influenza virus infectious cycle is onward transmission to new hosts. Environmental and physiological factors that differ between animal species may drive adaptations for transmission under different conditions. In humans, transmitted virions must access epithelial cells in the human respiratory mucosa where the $\mathrm{pH}$ can be as low as 5.5 (reviewed in $\mathrm{REF}^{148}$ ). This value is close to the $\mathrm{pH}$ at which HA is irreversibly triggered to undergo fusion; therefore, it could inactivate the virus prematurely in secretions outside the target cell or in respiratory droplets as they pass from one host to the next ${ }^{148,149}$ (FIG. 2C). Human seasonal influenza viruses that transmit efficiently through the air have HAs with lower fusion $\mathrm{pH}$ than those isolated from poultry or pigs ${ }^{150,151}$ (BOX 2; FIG. 4). A key change to H5N1 AIVs that enabled airborne transmission was a mutation that stabilized $\mathrm{HA}^{23,24,152}$. Furthermore, mutations in HA that reduced $\mathrm{pH}$ stability of the 2009 pandemic H1N1 virus abrogated airborne transmissibility between ferrets ${ }^{153}$. Mutations that affect HA stability map across the entire structure of the HA protein (reviewed in REF. ${ }^{148}$ ), but many occur in the stem region close to the fusion peptide or at the interface between the three subunits of the HA trimer. They presumably affect protein stability by affecting the interaction between HA monomers within the trimer or by affecting the local environment around important histidine residues that become protonated at low $\mathrm{pH}$ and stimulate the HA to extrude the fusion peptide ${ }^{154}$. The plethora of different mutations that can affect the $\mathrm{pH}$ of fusion of HA means that this phenotype is difficult to predict through sequence analysis. Rather, a phenotypic assay to measure HA fusion can be used ${ }^{148}$.

Under dense housing conditions, as in poultry houses or pig farms, environmental stability of the virion may have a smaller role in onward transmission than in circumstances in which hosts are more disperse. Thus, the intracellular fitness gain conferred by fusion in early rather than late endosomes drives selection of virus with higher $\mathrm{pH}$ of fusion. Uncoating in early endosomes might also allow viruses to evade restriction by ISGs such as the IFITM3 protein that inhibits virus fusion in later endosomal compartments ${ }^{78,155,156}$.

Thus, for both receptor binding and virion stability traits, human-adapting mutations may attenuate AIV pathogenicity. AIVs attach preferentially to cells expressing a2-3 SA in the lower airways and uncoat in early endosomes; therefore, they may be virulent but poorly transmissible. Human-adapted viruses that primarily target the a2-6-SA-rich upper airways and retain good airborne stability may be more transmissible but less virulent. This balance between virulence and transmissibility suggests that viruses that cross from poultry into humans would undergo some decrease in pathogenicity upon acquiring a transmissible phenotype, but this hypothesis has yet to be robustly tested.

\section{Conclusions and future perspectives}

Despite many transcriptomic and proteomic studies being conducted to identify host factors that are important for influenza virus replication, only a limited number of host factors are commonly identified between these screens, demonstrating inconsistencies and the

\section{Box 2 | The role of pigs in the adaptation of avian influenza viruses to humans}

It is a well-worn tenet of influenza virus biology that pigs are a 'mixing vessel' and key to the emergence of human pandemic influenza viruses. The idea is that pigs can be co-infected with an avian and a human influenza virus, and the reassortment events that can initiate a pandemic would occur within these animals.

Pigs are indeed susceptible to human-adapted viruses and to some avian influenza viruses (AIVs), as shown experimentally and observed in the field ${ }^{166,167}$. Swine influenza $A$ viruses that are currently enzootic in pigs are of the $\mathrm{H} 1$ and $\mathrm{H} 3$ haemagglutinin $(\mathrm{HA})$ subtypes that originate from various sources, including direct introduction from birds (for example, the Eurasian H1N1 lineage), re-introduction from humans (for example, pandemic H1N1 viruses) or following reassortment between human and swine viruses (for example, the variant $\mathrm{H} 3 \mathrm{~N} 2$ influenza $A$ viruses).

The mixing vessel concept has assumed that pigs are unique in being susceptible to infection by both avian and mammalian-adapted influenza strains, facilitating the process of antigenic shift by reassortment (BOX 1; FIC. 1).

However, from a physiological perspective, pigs are no more permissive to infection by AIVs than are humans: the type of sialic acid linkage that predominates in the swine upper respiratory tract is the $\alpha 2-6$ sialic acid as in humans ${ }^{28}$, and AIV replication is restricted in swine cells in the same manner as it is in human cells because swine acidic leucine-rich nuclear phosphoprotein 32 family member A (ANP32A) lacks the avian-specific gene duplication that supports activity of avian virus polymerase $\mathrm{e}^{106,168}$. Thus, the explanation of their prominent role in supporting influenza virus evolution may be more one of opportunity than physiology: the chances of close contact between infected domestic or wild birds and swine, and the sheer numbers and dense housing on pig farms allowing for close-contact transmission events between pigs, may create an environment more conducive to the evolution of viruses containing the adequate constellation of adaptive mutations to allow the avian-to-mammalian switch (FIG. 4). 


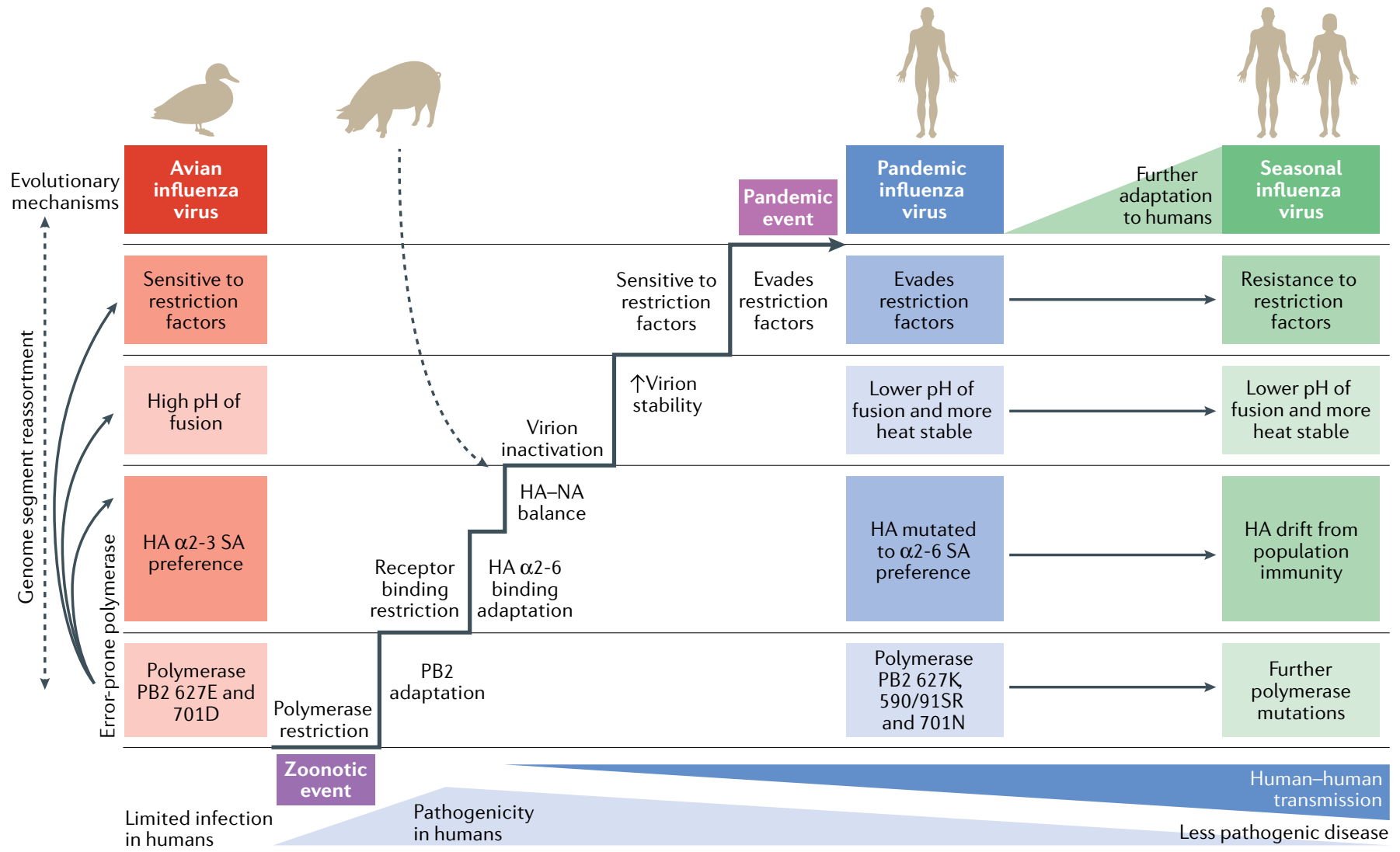

Fig. 4 | Cumulative influenza virus adaptations required to overcome species restriction and initiate a new pandemic. Influenza viruses circulating in the avian reservoir (red) face multiple host range barriers that must be overcome by genetic adaptation before a pandemic is initiated in humans (blue). Influenza viruses overcome host range barriers through their ability to undergo genetic reassortment (BOX 1) and the rapid generation of mutations by the error-prone viral RNA-dependent RNA polymerase. During a zoonotic infection, the restricted avian influenza virus (AIV) polymerase may acquire mutations in polymerase basic protein 2 (PB2) such as E627K or D701N. These mutations may cause severe disease in humans but no onward transmission without further mutation. Binding to $\alpha 2-6$ sialic acid (SA) receptors present on the cell surfaces of the human upper respiratory tract (URT) is enabled by mutations in the haemagglutinin (HA) receptor binding site but may require further mutations to address the balance between HA and neuraminidase (NA) activities. The diverse host range of influenza viruses (FIG. 1) may allow these adaptive steps via an intermediate mammalian species. For example, swine influenza A viruses acquire these first two adaptations, but further adaptation to humans are necessary (dotted line). For efficient human-human transmission, HA must be sufficiently stable to survive the $\mathrm{pH}$ of the human URT and airborne droplets. Antiviral antagonists in human cells such as interferon-regulated resistance GTP-binding protein MxA may drive selection of mutations in viral proteins such as NP. The fitness landscape of the virus may be altered at each adaptation step, limiting the likelihood of viruses reaching pandemic potential. After a pandemic event, further adaptation to humans occurs (green), which may moderate disease severity.

technical limitations of such studies ${ }^{157,158}$. Furthermore, the majority of these studies have not addressed the role of host proteins in species restriction.

With the advent of next-generation sequencing, it is now possible to identify within-host mutations and quantify selection bottlenecks to understand how host-adapting mutations are propagated ${ }^{159,160}$. This knowledge may help us better understand why some AIVs cross the species barrier and why others do not.

Most cross-species incursions result in dead-end infections because the virus does not accumulate sufficient adaptive changes quickly enough in the new host, particularly if the mutations must be acquired in a particular order to avoid intermediates with fitness costs ${ }^{161}$. An onward chain of transmission is not supported until a sufficient constellation of adaptive changes that affect several features of the virus-host interaction are acquired (FIG. 4). It is not clear whether some of the mutations could be acquired animal side or whether all are selected in the new host after the zoonotic infection. Bearing in mind the viral mutation rate, replication rate and limited time window for onward transmission, the likelihood that a complete set of adaptive mutations is acquired in a single immunocompetent host and transmitted onwards to other individuals is extremely low ${ }^{162,163}$.

Understanding the mechanisms that underlie the adaptive changes and the circumstances in which they can be acquired will enhance our ability to predict the source and nature of future pandemics. It will also reveal intricate details of virus-host interactions that can lead to novel therapeutics and antiviral strategies.

Published online 28 November 2018 
1. Zhai, S.-L. et al. Influenza D virus in animal species in Guangdong province, Southern China. Emerg. Infect. Dis. 23, 1392-1396 (2017)

2. Webster, R. G., Yakhno, M., Hinshaw, V. S., Bean, W. J. \& Murti, K. G. Intestinal influenza: replication and characterization of influenza viruses in ducks. Virology 84, 268-278 (1978).

3. Ciminski, K., Thamamongood, T., Zimmer, G. \& Schwemmle, M. Novel insights into bat influenza A viruses. J. Gen. Virol. 98, 2393-2400 (2017).

4. Taubenberger, J. K. \& Kash, J. C. Influenza virus evolution, host adaptation, and pandemic formation. Cell Host Microbe 7, 440-451 (2010).

5. Bouvier, N. M. Animal models for influenza virus transmission studies: a historical perspective. Curr. Opin. Virol. 13, 101-108 (2015)

6. Belser, J. A., Eckert, A. M., Tumpey, T. M. \& Maines, T. R. Complexities in ferret influenza virus pathogenesis and transmission models. Microbiol. Mol. Biol. Rev. 80, 733-744 (2016)

7. O'Donnell, C. D. \& Subbarao, K. The contribution of animal models to the understanding of the host range and virulence of influenza A viruses. Microbes Infect. 13, 502-515 (2011).

8. Varki, A. Colloquium paper: uniquely human evolution of sialic acid genetics and biology. Proc. Natl Acad. Sci. USA 107 (Suppl. 2), 8939-8946 (2010).

9. Okerblom, J. J. et al. Loss of CMAH during human evolution primed the monocyte-macrophage lineage toward a more inflammatory and phagocytic state. J. Immunol. 198, 2366-2373 (2017).

10. Naito-Matsui, Y. et al. Physiological exploration of the long term evolutionary selection against expression of $\mathrm{N}$-glycolylneuraminic acid in the brain. J. Biol. Chem. 292, 2557-2570 (2017).

11. Ng, P. S. K. et al. Ferrets exclusively synthesize Neu5Ac and express naturally humanized influenza A virus receptors. Nat. Commun. 5, 5750 (2014).

12. Suzuki, Y. et al. Sialic acid species as a determinant of the host range of influenza A viruses. J. Virol. 74, 11825-11831 (2000).

13. Rogers, G. N. \& Paulson, J. C. Receptor determinants of human and animal influenza virus isolates: differences in receptor specificity of the $\mathrm{H} 3$ hemagglutinin based on species of origin. Virology 127, 361-373 (1983).

14. Rogers, G. N. et al. Single amino acid substitutions in influenza haemagglutinin change receptor binding specificity. Nature 304, 76-78 (1983). This seminal study reveals the amino acids of the HA binding site that recognize the different linkages of SA that predominate in human or avian hosts.

15. Shi, Y., Wu, Y., Zhang, W., Qi, J. \& Gao, G. F. Enabling the 'host jump': structural determinants of receptorbinding specificity in influenza A viruses. Nat. Rev. Microbiol. 12, 822-831 (2014).

16. Lipsitch, M. et al. Viral factors in influenza pandemic risk assessment. eLife 5, e18491 (2016).

17. Chandrasekaran, A. et al. Glycan topology determines human adaptation of avian $\mathrm{H} 5 \mathrm{~N} 1$ virus hemagglutinin Nat. Biotechnol. 26, 107-113 (2008).

18. Matrosovich, M. et al. Early alterations of the receptor-binding properties of $\mathrm{H} 1, \mathrm{H} 2$, and $\mathrm{H} 3$ avian influenza virus hemagglutinins after their introduction into mammals. J. Virol. 74, 8502-8512 (2000).

19. Pappas, C. et al. Receptor specificity and transmission of $\mathrm{H} 2 \mathrm{~N} 2$ subtype viruses isolated from the pandemic of 1957. PLOS ONE 5, e11158 (2010).

20. Tumpey, T. M. et al. A two-amino acid change in the hemagglutinin of the 1918 influenza virus abolishes transmission. Science 315, 655-659 (2007).

The 1918 influenza pandemic virus is reconstructed and two amino acids in the RBS are shown to determine the transmissibility of the virus through the air between ferrets. This is the first study to use the ferret model in this way to demonstrate the molecular determinants of transmissibility.

21. Roberts, K. L., Shelton, H., Scull, M., Pickles, R. \& Barclay, W. S. Lack of transmission of a human influenza virus with avian receptor specificity between ferrets is not due to decreased virus shedding but rather a lower infectivity in vivo. J. Gen. Virol. 92, 1822-1831 (2011)

22. Xiong, X. et al. Receptor binding by an H7N9 influenza virus from humans. Nature 499, 496-499 (2013).

23. Imai, M. et al. Experimental adaptation of an influenza H5 HA confers respiratory droplet transmission to a reassortant $\mathrm{H} 5 \mathrm{HA} / \mathrm{H} 1 \mathrm{~N} 1$ virus in ferrets. Nature $\mathbf{4 8 6}$ 420-428 (2012)

Using a reassortant AIV with H5 HA, this study identifies mutations that are required for airborne transmission of avian $\mathrm{H} 5$ subtype viruses.
24. Herfst, S. et al. Airborne transmission of influenza A/H5N 1 virus between ferrets. Science 336

1534-1541 (2012)

By passaging recombinant $\mathrm{H} 5 \mathrm{~N} 1$ influenza viruses between ferrets, this study shows that an H5N1 AIV could transmit through the air and details mutations that enable the virus to do so, including the necessity for stabilization of $\mathrm{HA}$

25. Shinya, K. et al. Avian flu: influenza virus receptors in the human airway. Nature 440, 435-436 (2006).

26. Lakdawala, S. S. et al. The soft palate is an important site of adaptation for transmissible influenza viruses. Nature 526, 122-125 (2015)

27. Walther, T. et al. Glycomic analysis of human respiratory tract tissues and correlation with influenza virus infection. PLOS Pathog. 9, e1003223 (2013).

28. Nelli, R. K. et al. Comparative distribution of human and avian type sialic acid influenza receptors in the pig. BMC Vet. Res. 6, 4 (2010)

29. Jia, N. et al. Glycomic characterization of respiratory tract tissues of ferrets: implications for its use in influenza virus infection studies. J. Biol. Chem. 289 28489-28504 (2014)

30. Ning, Z.-Y. et al. Detection of expression of influenza virus receptors in tissues of BALB/c mice by histochemistry. Vet. Res. Commun. 33, 895-903 (2009).

31. Gagneux, P. et al. Human-specific regulation of alpha 2-6-linked sialic acids. J. Biol. Chem. 278 48245-48250 (2003).

32. van Riel, D. et al. H5N1 virus attachment to lower respiratory tract. Science 312, 399 (2006)

33. Srinivasan, A. et al. Quantitative biochemical rational for differences in transmissibility of 1918 pandemic influenza A viruses. Proc. Natl Acad. Sci. USA 105, 2800-2805 (2008).

34. Costa, T. et al. Distribution patterns of influenza virus receptors and viral attachment patterns in the respiratory and intestinal tracts of seven avian species. Vet. Res. 43, 28 (2012)

35. Kuchipudi, S. V. et al. Differences in influenza virus receptors in chickens and ducks: implications for interspecies transmission. J. Mol. Genet. Med. 3 , 143-151 (2009)

36. Kimble, B., Nieto, G. R. \& Perez, D. R. Characterization of influenza virus sialic acid receptors in minor poultry species. Virol. J. 7, 365 (2010).

37. Gujjar, N. et al. Co-expression of sialic acid receptors compatible with avian and human influenza virus binding in emus (Dromaius novaehollandiae). Virology 500, 114-121 (2017).

38. Chan, R. W. Y. et al. Infection of swine ex vivo tissues with avian viruses including H7N9 and correlation with glycomic analysis. Influenza Other Respir. Viruses 7, 1269-1282 (2013).

39. Bateman, A. C. et al. Glycan analysis and influenza A virus infection of primary swine respiratory epithelial cells: the importance of NeuAca 2-6 glycans. J. Biol. Chem. 285, 34016-34026 (2010).

40. Byrd-Leotis, L. et al. Shotgun glycomics of pig lung identifies natural endogenous receptors for influenza viruses. Proc. Natl Acad. Sci. USA 111, E2241-E2250 (2014).

41. Peng, W. et al. Recent H3N2 viruses have evolved specificity for extended, branched human-type receptors, conferring potential for increased avidity. Cell Host Microbe 21, 23-34 (2017).

42. Heaton, B. E. et al. A CRISPR activation screen identifies a pan-avian influenza virus inhibitory host factor. Cell Rep. 20, 1503-1512 (2017).

43. Hoffmann, T. W. et al. Length variations in the NA stalk of an $\mathrm{H} 7 \mathrm{~N} 1$ influenza virus have opposite effects on viral excretion in chickens and ducks. J. Virol. 86 584-588 (2012)

44. Blumenkrantz, D., Roberts, K. L., Shelton, H., Lycett, S. $\&$ Barclay, W. S. The short stalk length of HPAI H5N1 influenza neuraminidase limits transmission of pandemic $\mathrm{H} 1 \mathrm{~N} 1$ virus in ferrets. J. Virol. 87 10539-10551 (2013).

45. $\mathrm{Xu}, \mathrm{R}$. et al. Functional balance of the hemagglutinin and neuraminidase activities accompanies the emergence of the $2009 \mathrm{H} 1 \mathrm{~N} 1$ influenza pandemic. J. Virol. 86, 9221-9232 (2012).

46. Yen, H.-L. et al. Hemagglutinin-neuraminidase balance confers respiratory-droplet transmissibility of the pandemic $\mathrm{H} 1 \mathrm{~N} 1$ influenza virus in ferrets. Proc. Natl Acad. Sci. USA 108, 14264-14269 (2011).

47. Lakdawala, S. S. et al. Eurasian-origin gene segments contribute to the transmissibility, aerosol release, and morphology of the 2009 pandemic H1N1 influenza virus. PLOS Pathog. 7, e1002443 (2011).
48. Uhlendorff, J., Matrosovich, T., Klenk, H.-D. \& Matrosovich, M. Functional significance of the hemadsorption activity of influenza virus neuraminidase and its alteration in pandemic viruses. Arch. Virol. 154, 945-957 (2009).

49. Benton, D. J., Wharton, S. A., Martin, S. R. \& McCauley, J. W. Role of neuraminidase in influenza $\mathrm{A}(\mathrm{H} 7 \mathrm{~N} 9)$ virus receptor binding. J. Virol. 91 , e02293-16 (2017)

50. Edinger, T. O., Pohl, M. O. \& Stertz, S. Entry of influenza A virus: host factors and antiviral targets. J. Gen. Virol. 95, 263-277 (2014).

51. Flatt, J. W. \& Greber, U. F. Misdelivery at the nuclear pore complex - stopping a virus dead in its tracks. Cells 4, 277-296 (2015).

52. Rehwinkel, J. et al. RIG-I detects viral genomic RNA during negative-strand RNA virus infection. Cell 140 397-408 (2010)

53. Meyerson, N. R. et al. Nuclear TRIM25 specifically targets influenza virus ribonucleoproteins to block the onset of RNA chain elongation. Cell Host Microbe 22 627-638 (2017).

54. Weber, M. et al. Influenza virus adaptation PB2-627K modulates nucleocapsid inhibition by the pathogen sensor RIG-I. Cell Host Microbe 17, 309-319 (2015).

55. Rameix-Welti, M.-A., Tomoiu, A., Dos Santos Afonso, E. van der Werf, S. \& Naffakh, N. Avian Influenza A virus polymerase association with nucleoprotein, but not polymerase assembly, is impaired in human cells during the course of infection. J. Virol. 83 1320-1331 (2009).

56. Mehle, A. \& Doudna, J. A. An inhibitory activity in human cells restricts the function of an avian-like influenza virus polymerase. Cell Host Microbe 4, 111-122 (2008)

57. Barber, M. R. W., Aldridge, J. R., Webster, R. G \& Magor, K. E. Association of RIG-I with innate immunity of ducks to influenza. Proc. Natl Acad. Sci. USA 107, 5913-5918 (2010)

58. Shao, Q. et al. RIG-I from waterfowl and mammals differ in their abilities to induce antiviral responses against influenza A viruses. J. Gen. Virol. 96, 277-287 (2015).

59. Long, J. S. et al. The effect of the PB2 mutation $627 \mathrm{~K}$ on highly pathogenic H5N 1 avian influenza virus is dependent on the virus lineage. J. Virol. 87 9983-9996 (2013).

60. Cauldwell, A. V., Moncorgé, O. \& Barclay, W. S Unstable polymerase-nucleoprotein interaction is not responsible for avian influenza virus polymerase restriction in human cells. J. Virol. 87, 1278-1284 (2013).

61. Staeheli, P., Haller, O., Boll, W., Lindenmann, J. \& Weissmann, C. Mx protein: constitutive expression in $3 \mathrm{~T} 3$ cells transformed with cloned Mx cDNA confers selective resistance to influenza virus. Cell 44, 147-158 (1986)

This article presents the first description of a host factor that confers resistance to influenza virus.

62. Haller, O., Staeheli, P., Schwemmle, M. \& Kochs, G. Mx GTPases: dynamin-like antiviral machines of innate immunity. Trends Microbiol. 23, 154-163 (2015).

63. Xiao, H., Killip, M. J., Staeheli, P., Randall, R. E. $\Sigma$ Jackson, D. The human interferon-induced MxA protein inhibits early stages of influenza $A$ virus infection by retaining the incoming viral genome in the cytoplasm. J. Virol. 87, 13053-13058 (2013).

64. Dornfeld, D. et al. SMARCA2-regulated host cell factors are required for MxA restriction of influenza A viruses. Sci. Rep. 8, 2092 (2018)

65. Benfield, C. T. O., Lyall, J. W., Kochs, G. \& Tiley, L. S. Asparagine 631 variants of the chicken Mx protein do not inhibit influenza virus replication in primary chicken embryo fibroblasts or in vitro surrogate assays. J. Virol. 82, 7533-7539 (2008).

66. Mänz, B. et al. Pandemic influenza A viruses escape from restriction by human $M \times A$ through adaptive mutations in the nucleoprotein. PLOS Pathog. 9, e 1003279 (2013)

This paper provides a description of mutations in a viral protein that allow evasion of the $\mathrm{Mx}$ restriction factor and are linked with the emergence of pandemic influenza viruses from AIV in humans.

67. Dittmann, J. et al. Influenza A virus strains differ in sensitivity to the antiviral action of Mx-GTPase J. Virol. 82, 3624-3631 (2008).

68. Ashenberg, O., Padmakumar, J., Doud, M. B. \& Bloom, J. D. Deep mutational scanning identifies sites in influenza nucleoprotein that affect viral inhibition by MxA. PLOS Pathog. 13, e 1006288 (2017). 
69. Deeg, C. M. et al. In vivo evasion of MxA by avian influenza viruses requires human signature in the viral nucleoprotein. J. Exp. Med. 214, 1239-1248 (2017).

70. Götz, V. et al. Influenza A viruses escape from MxA restriction at the expense of efficient nuclear vRNP import. Sci. Rep. 6, 23138 (2016).

71. Riegger, D. et al. The nucleoprotein of newly emerged H7N9 influenza A virus harbors a unique motif conferring resistance to antiviral human MxA. J. Virol. 89, 2241-2252 (2015)

72. Feeley, E. M. et al. IFITM3 inhibits influenza A virus infection by preventing cytosolic entry. PLOS Pathog 7, e1002337 (2011).

73. Chen, G., Liu, C.-H., Zhou, L. \& Krug, R. M. Cellular DDX21 RNA helicase inhibits influenza A virus replication but is counteracted by the viral NS 1 protein. Cell Host Microbe 15, 484-493 (2014).

74. Zhang, J. et al. Host protein Moloney Leukemia Virus 10 (MOV10) acts as a restriction factor of influenza A virus by inhibiting the nuclear import of the viral nucleoprotein J. Virol. 90, 3966-3980 (2016).

75. Nordmann, A., Wixler, L., Boergeling, Y., Wixler, V. \& Ludwig, S. A new splice variant of the human guanylate-binding protein 3 mediates anti-influenza activity through inhibition of viral transcription and replication. FASEB J. 26, 1290-1300 (2012).

76. Hsu, W.-B. et al. Cellular protein HAX1 interacts with the influenza A virus PA polymerase subunit and impedes its nuclear translocation. J. Virol. 87, 110-123 (2013)

77. Di Pietro, A. et al. TRIM22 inhibits influenza A virus infection by targeting the viral nucleoprotein for degradation. J. Virol. 87, 4523-4533 (2013).

78. Gerlach, T. et al. pH optimum of hemagglutinin-mediated membrane fusion determines sensitivity of influenza A viruses to the interferon-induced antiviral state and IFITMs. J. Virol. 91, e00246-17 (2017).

79. Pagani, I. et al. Mutations conferring increased sensitivity to tripartite motif 22 restriction accumulated progressively in the nucleoprotein of seasonal influenza A ( $\mathrm{H} 1 \mathrm{~N} 1)$ viruses between 1918 and 2009. mSphere 3, e00110-18 (2018).

80. Mazel-Sanchez, B., Boal Carvalho, I., Silva, F. Dijkman, R. \& Schmolke, M. H5N1 influenza A virus PB 1-F2 relieves HAX-1-mediated restriction of avian virus polymerase PA in human lung cells. J. Virol. 92 e00425-18 (2018)

81. O'Neill, R. E. \& Palese, P. NPI-1, the human homolog of SRP-1, interacts with influenza virus nucleoprotein. Virology 206, 116-125 (1995).

82. Resa-Infante, P. \& Gabriel, G. The nuclear import machinery is a determinant of influenza virus host adaptation. Bioessays 35, 23-27 (2013).

83. Czudai-Matwich, V., Otte, A., Matrosovich, M., Gabriel, G. \& Klenk, H.-D. PB2 mutations D701N and S714R promote adaptation of an influenza H5N 1 virus to a mammalian host. J. Virol. 88, 8735-8742 (2014).

84. Gabriel, G., Herwig, A. \& Klenk, H.-D. Interaction of polymerase subunit PB2 and NP with importin alpha1 is a determinant of host range of influenza $A$ virus. PLOS Pathog. 4, e11 (2008)

85. Sediri, H., Schwalm, F., Gabriel, G. \& Klenk, H.-D. Adaptive mutation PB2 D701N promotes nuclear import of influenza vRNPs in mammalian cells. Eur. J. Cell Biol. 94, 368-374 (2015). This study demonstrates the importance of specific members of the importin- $\alpha$ family of host factors in determining activity in the human cell nucleus of AIV or human-adapted polymerase.

86. Tarendeau, F. et al. Structure and nuclear import function of the C-terminal domain of influenza virus polymerase PB2 subunit. Nat. Struct. Mol. Biol. 14 229-233 (2007)

87. Boivin, S. \& Hart, D. J. Interaction of the influenza A virus polymerase PB2 C-terminal region with importin alpha isoforms provides insights into host adaptation and polymerase assembly. J. Biol. Chem 286, 10439-10448 (2011).

88. Resa-Infante, P. et al. The host-dependent interaction of a-importins with influenza PB2 polymerase subunit is required for virus RNA replication. PLOS ONE 3 e3904 (2008).

89. Gabriel, G. et al. Differential use of importin- $\alpha$ isoforms governs cell tropism and host adaptation of influenza virus. Nat. Commun. 2, 156 (2011)

90. Hudjetz, B. \& Gabriel, G. Human-like PB2 627K influenza virus polymerase activity is regulated by importin- 1 and - $\alpha$ 7. PLOS Pathog. 8, e1002488 (2012).
91. Ninpan, K. et al. Expression of importin- $\alpha$ isoforms in human nasal mucosa: implication for adaptation of avian influenza A viruses to human host. Virol. J. 13 90 (2016)

92. Te Velthuis, A. J. W. \& Fodor, E. Influenza virus RNA polymerase: insights into the mechanisms of viral RNA synthesis. Nat. Rev. Microbiol. 14, 479-493 (2016).

93. Eisfeld, A. J., Neumann, G. \& Kawaoka, Y. At the centre: influenza A virus ribonucleoproteins. Nat. Rev. Microbiol. 13, 28-41 (2015).

94. Mänz, B., Brunotte, L., Reuther, P. \& Schwemmle, M. Adaptive mutations in NEP compensate for defective H5N1 RNA replication in cultured human cells. Nat. Commun. 3, 802 (2012).

This study reveals which step in the replication of an AIV polymerase is restricted in mammalian cells and uncovers a role for NEP in host adaptation.

95. Cauldwell, A. V., Long, J. S., Moncorgé, O. \& Barclay, W. S. Viral determinants of influenza A host range. J. Gen. Virol. 95, 1193-1210 (2014).

96. Subbarao, E. K., London, W. \& Murphy, B. R. A single amino acid in the PB2 gene of influenza $A$ virus is a determinant of host range. J. Virol. 67, 1761-1764 (1993).

This study identifies the PB2 E627K mutation as a determinant of host range for the first time.

97. Taubenberger, J. K. et al. Characterization of the 1918 influenza virus polymerase genes. Nature 437, 889-893 (2005)

98. Mehle, A. \& Doudna, J. A. Adaptive strategies of the influenza virus polymerase for replication in humans. Proc. Natl Acad. Sci. USA 106, 21312-21316 (2009).

99. Pflug, A., Guilligay, D., Reich, S. \& Cusack, S. Structure of influenza A polymerase bound to the viral RNA promoter. Nature 516, 355-360 (2014).

100. Yamada, S. et al. Biological and structural characterization of a host-adapting amino acid in influenza virus. PLOS Pathog. 6, e 1001034 (2010).

101. Tarendeau, F. et al. Host determinant residue lysine 627 lies on the surface of a discrete, folded domain of influenza virus polymerase PB2 subunit. PLOS Pathog. 4, e1000136 (2008)

102. Massin, P., van der Werf, S. \& Naffakh, N. Residue 627 of PB2 is a determinant of cold sensitivity in RNA replication of avian influenza viruses. J. Virol. 75, 5398-5404 (2001).

103. Aggarwal, S., Dewhurst, S., Takimoto, T. \& Kim, B. Biochemical impact of the host adaptation associated PB2 E627K mutation on the temperature-dependent RNA synthesis kinetics of influenza A virus polymerase complex. J. Biol. Chem. 286, 34504-34513 (2011).

104. Luytjes, W., Krystal, M., Enami, M., Parvin, J. D. ¿ Palese, P. Amplification, expression, and packaging of foreign gene by influenza virus. Cell 59, 1107-1113 (1989).

105. Moncorgé, O., Mura, M. \& Barclay, W. S. Evidence for avian and human host cell factors that affect the activity of influenza virus polymerase. J. Virol. 84 9978-9986 (2010).

106. Long, J. S. et al. Species difference in ANP32A underlies influenza A virus polymerase host restriction. Nature 529, 101-104 (2016). This study describes the discovery of the host factor that underlies avian influenza $A$ virus polymerase restriction in human cells.

107. Reilly, P. T., Yu, Y., Hamiche, A. \& Wang, L. Cracking the ANP32 whips: important functions, unequal requirement, and hints at disease implications. Bioessays 36, 1062-1071 (2014).

108. Watanabe, T et al. Influenza virus-host interactome screen as a platform for antiviral drug development. Cell Host Microbe 16, 795-805 (2014).

109. Sugiyama, K., Kawaguchi, A., Okuwaki, M. \& Nagata, K pp32 and APRIL are host cell-derived regulators of influenza virus RNA synthesis from CRNA. eLife 4, e08939 (2015)

110. Domingues, P. \& Hale, B. G. Functional insights into ANP32A-dependent influenza A virus polymerase hos restriction. Cell Rep. 20, 2538-2546 (2017)

111. Baker, S. F., Ledwith, M. P. \& Mehle, A. Differential splicing of ANP32A in birds alters its ability to stimulate RNA synthesis by restricted influenza polymerase. Cell Rep. 24, 2581-2588 (2018)

112. Shinya, K. et al. Ostrich involvement in the selection of $\mathrm{H} 5 \mathrm{~N} 1$ influenza virus possessing mammalian-type amino acids in the PB2 protein. J. Virol. $\mathbf{8 3}$, 13015-13018 (2009).

113. Bradel-Tretheway, B. G. et al. Comprehensive proteomic analysis of influenza virus polymerase complex reveals a novel association with mitochondrial proteins and RNA polymerase accessory factors. J. Virol. 85, 8569-8581 (2011)

114. Hengrung, N. et al. Crystal structure of the RNA-dependent RNA polymerase from influenza C virus. Nature 527, 114-117 (2015).

115. Chang, S. et al. Cryo-EM structure of influenza virus RNA polymerase complex at $4.3 \AA$ resolution. Mol. Cell 57, 925-935 (2015).

116. Thierry, E. et al. Influenza polymerase can adopt an alternative configuration involving a radical repacking of PB2 domains. Mol. Cell 61, 125-137 (2016).

117. Neumann, G. \& Hobom, G. Mutational analysis of influenza virus promoter elements in vivo. J. Gen. Virol. 76, 1709-1717 (1995).

118. Crescenzo-Chaigne, B., van der Werf, S. \& Naffakh, N. Differential effect of nucleotide substitutions in the $3^{\prime}$ arm of the influenza A virus vRNA promoter on transcription/replication by avian and human polymerase complexes is related to the nature of PB2 amino acid 627. Virology 303, 240-252 (2002)

119. Paterson, D., te Velthuis, A. J. W., Vreede, F. T. \& Fodor, E. Host restriction of influenza virus polymerase activity by PB2 627E is diminished on short viral templates in a nucleoprotein-independent manner. J. Virol. 88, 339-344 (2014).

120. Nilsson, B. E., Te Velthuis, A. J. W. \& Fodor, E. Role of the PB2 627 domain in influenza A virus polymerase function. J. Virol. 91, e02467-16 (2017).

121. Bortz, E. et al. Host- and strain-specific regulation of influenza virus polymerase activity by interacting cellular proteins. mBio 2, e00151-11 (2011).

122. Rodriguez-Frandsen, A., Alfonso, R. \& Nieto, A. Influenza virus polymerase: functions on host range, inhibition of cellular response to infection and pathogenicity. Virus Res. 209, 23-38 (2015).

123. Mehle, A., Dugan, V. G., Taubenberger, J. K. \& Doudna, J. A. Reassortment and mutation of the avian influenza virus polymerase PA subunit overcome species barriers. J. Virol. 86, 1750-1757 (2012)

124. Zhou, B. et al. PB2 residue 158 is a pathogenic determinant of pandemic $\mathrm{H} 1 \mathrm{~N} 1$ and $\mathrm{H} 5$ influenza a viruses in mice. J. Virol. 85, 357-365 (2011).

125. Hayashi, T., Wills, S., Bussey, K. A. \& Takimoto, T. Identification of influenza A virus PB2 residues involved in enhanced polymerase activity and virus growth in mammalian cells at low temperatures. J. Virol. 89, 8042-8049 (2015).

126. Robb, N. C., Smith, M., Vreede, F. T. \& Fodor, E. NS2 NEP protein regulates transcription and replication of the influenza virus RNA genome. J. Gen. Virol. 90, 1398-1407 (2009).

127. Reuther, P. et al. Adaptive mutations in the nuclear export protein of human-derived H5N 1 strains facilitate a polymerase activity-enhancing conformation. J. Virol. 88, 263-271 (2014).

128. Perez, J. T. et al. A small RNA enhancer of viral polymerase activity. J. Virol. 86, 13475-13485 (2012).

129. Perez, J. T. et al. Influenza A virus-generated small RNAs regulate the switch from transcription to replication. Proc. Natl Acad. Sci. USA 107 11525-11530 (2010).

130. Brunotte, L. et al. The nuclear export protein of $\mathrm{H} 5 \mathrm{~N}$ influenza A viruses recruits Matrix 1 (M1) protein to the viral ribonucleoprotein to mediate nuclear export. J. Biol. Chem. 289, 20067-20077 (2014).

131. Killip, M. J., Jackson, D., Pérez-Cidoncha, M., Fodor, E. $\&$ Randall, R. E. Single-cell studies of IFN- $\beta$ promoter activation by wild-type and NS1-defective influenza A viruses. J. Gen. Virol. 98, 357-363 (2017).

132. Chiang, J. J. et al. Viral unmasking of cellular $5 S$ rRNA pseudogene transcripts induces RIG-I-mediated immunity. Nat. Immunol. 19, 53-62 (2018).

133. Hayman, a et al. NS1 proteins of avian influenza A viruses can act as antagonists of the human alpha/ beta interferon response. J. Virol. 81, 2318-2327 (2007).

134. Turnbull, M. L. et al. Role of the B allele of influenza A virus segment 8 in setting mammalian host range and pathogenicity. J. Virol. 90, 9263-9284 (2016).

135. Hale, B. G. et al. Inefficient control of host gene expression by the 2009 pandemic H1N1 influenza A virus NS1 protein. J. Virol. 84, 6909-6922 (2010).

136. Twu K. Y Kuo, R.-L., Marklund, J \& Krug R. M. The H5N 1 influenza virus NS genes selected after 1998 enhance virus replication in mammalian cells. J. Virol. 81, 8112-8121 (2007)

137. Chauché, C. et al. Mammalian adaptation of an avian influenza A virus involves stepwise changes in NS1. J. Virol. 92, e01875-17 (2018).

138. Tsai, P.-L. et al. Cellular RNA binding proteins NS1-BP and hnRNP K regulate influenza A virus RNA splicing. PLOS Pathog. 9, e1003460 (2013). 
139. Selman, M., Dankar, S. K., Forbes, N. E., Jia, J.-J. $\propto$ Brown, E. G. Adaptive mutation in influenza A virus non-structural gene is linked to host switching and induces a novel protein by alternative splicing. Emerg. Microbes Infect. 1, e42 (2012).

140. Huang, X. et al. An NS-segment exonic splicing enhancer regulates influenza A virus replication in mammalian cells. Nat. Commun. 8, 14751 (2017).

141. Beale, R. et al. A LC3-interacting motif in the influenza $A$ virus $M 2$ protein is required to subvert autophagy and maintain virion stability. Cell Host Microbe 15, 239-247 (2014)

142. Kuo, S.-M. et al. Inhibition of avian influenza A virus replication in human cells by host restriction factor TUFM is correlated with autophagy. $m B i o \mathbf{8}$, e00481-17 (2017)

143. Graef, K. M. et al. The PB2 subunit of the influenza virus RNA polymerase affects virulence by interacting with the mitochondrial antiviral signaling protein and inhibiting expression of beta interferon. J. Virol. 84 8433-8445 (2010)

144. Varga, Z. T. et al. The influenza virus protein PB1-F2 inhibits the induction of type I interferon at the level of the MAVS adaptor protein. PLOS Pathog. 7, e 1002067 (2011).

145. Chen, W. et al. A novel influenza A virus mitochondrial protein that induces cell death. Nat. Med. 7 , 1306-1312 (2001)

146. James, J. et al. Influenza A virus PB1-F2 protein prolongs viral shedding in chickens lengthening the transmission window. J. Gen. Virol. 97, 2516-2527 (2016).

147. Peiris, J. S. M., Cheung, C. Y., Leung C. Y. H. \& Nicholls, J. M. Innate immune responses to influenza A H5N 1: friend or foe? Trends Immunol. 30, 574-584 (2009).

148. Russell, C. J., Hu, M. \& Okda, F. A. Influenza hemagglutinin protein stability, activation, and pandemic risk. Trends Microbiol. 26, 841-853 (2018)

149. Yang, W. \& Marr, L. C. Mechanisms by which ambient humidity may affect viruses in aerosols. Appl. Environ. Microbiol. 78, 6781-6788 (2012).

150. Baumann, J., Kouassi, N. M., Foni, E., Klenk, H.-D. \& Matrosovich, M. H1N1 swine influenza viruses differ from avian precursors by a higher $\mathrm{pH}$ optimum of membrane fusion. J. Virol. 90, 1569-1577 (2015)

151. Galloway, S. E., Reed, M. L., Russell, C. J. \& Steinhauer, D. A. Influenza HA subtypes demonstrate divergent phenotypes for cleavage activation and $\mathrm{pH}$ of fusion: implications for host range and adaptation. PLOS Pathog. 9, e1003151 (2013).

152. Linster, M. et al. Identification, characterization, and natural selection of mutations driving airborne transmission of $\mathrm{A} / \mathrm{H} 5 \mathrm{~N} 1$ virus. Cell 157, 329-339 (2014).

153. Russier, M. et al. Molecular requirements for a pandemic influenza virus: an acid-stable hemagglutinin protein. Proc. Natl Acad. Sci. USA 113, 1636-1641 (2016).

154. Bullough, P. A., Hughson, F. M., Skehel, J. J. \& Wiley, D. C. Structure of influenza haemagglutinin at the $\mathrm{pH}$ of membrane fusion. Nature $371,37-43$ (1994).
155. Long, J. S., Benfield, C. T. \& Barclay, W. S. One-way trip: influenza virus' adaptation to gallinaceous poultry may limit its pandemic potential. Bioessays 37 204-212 (2015)

156. Sun, X. et al. Constitutively expressed IFITM3 protein in human endothelial cells poses an early infection block to human influenza viruses. J. Virol 90 11157-11167 (2016)

157. Chou, Y.-C. et al. Variations in genome-wide RNAi screens: lessons from influenza research. J. Clin. Bioinforma. 5, 2 (2015).

158. Powell, J. D. \& Waters, K. M. Influenza-omics and the host response: recent advances and future prospects. Pathogens 6, 25 (2017)

159. Zaraket, H. et al. Mammalian adaptation of influenza $\mathrm{A}(\mathrm{H} 7 \mathrm{~N} 9)$ virus is limited by a narrow genetic bottleneck. Nat Commun 6,6553 (2015).

160. Moncla, L. H. et al. Selective bottlenecks shape evolutionary pathways taken during mammalian adaptation of a 1918-like avian influenza virus. Cell Host Microbe 19, 169-180 (2016).

161. Illingworth, C. J. R. Fitness inference from short-read data: within-host evolution of a reassortant $\mathrm{H} 5 \mathrm{~N} 1$ influenza virus. Mol. Biol. Evol. 32, 3012-3026 (2015).

162. Russell, C. A. et al. The potential for respiratory droplet-transmissible $\mathrm{A} / \mathrm{H} 5 \mathrm{~N} 1$ influenza virus to evolve in a mammalian host. Science 336, 1541-1547 in a mam 2012 ).

This study uses a modelling approach to assess the likelihood that H5N1 AIV can acquire the set of mutations necessary to adapt the virus for airborne transmission between mammals.

163. Roberts, K. L., Shelton, H., Stilwell, P. \& Barclay, W. S Transmission of a $2009 \mathrm{H} 1 \mathrm{~N} 1$ pandemic influenza virus occurs before fever is detected, in the ferret model. PLOS ONE 7, e43303 (2012).

164. Chen, Y. et al. Emergence and evolution of nove reassortant influenza A viruses in canines in Southern China. mBio 9, e00909-18 (2018).

165. Reid, A. H., Taubenberger, J. K. \& Fanning, T. G. Evidence of an absence: the genetic origins of the 1918 pandemic influenza virus. Nat. Rev. Microbiol. 2, 909-914 (2004).

166. Kida, H. et al. Potential for transmission of avian influenza viruses to pigs. J. Gen. Virol. 75 2183-2188 (1994).

167. Vincent, A. et al. Review of influenza A virus in swine worldwide: a call for increased surveillance and research. Zoonoses Public Health 61, 4-17 (2014).

168. Moncorgé, O. et al. Investigation of influenza virus polymerase activity in pig cells. J. Virol. 87, 384-394 (2013).

169. Tong, S. et al. A distinct lineage of influenza A virus from bats. Proc. Natl Acad. Sci. USA 109, 4269-4274 (2012).

170. Osterhaus, A. D., Rimmelzwaan, G. F., Martina, B. E., Bestebroer, T. M. \& Fouchier, R. A. Influenza B virus in seals. Science 288, 1051-1053 (2000)

171. Hause, B. M. et al. Isolation of a novel swine influenza virus from Oklahoma in 2011 which is distantly related to human influenza C viruses. PLOS Pathog. 9, e 1003176 (2013)

172. Shi, M. et al. The evolutionary history of vertebrate RNA viruses. Nature 556, 197-202 (2018).

173. McFadden, E. R. et al. Thermal mapping of the airways in humans. J. Appl. Physiol. 58, 564-570 (1985).

174. Labadie, K., Dos Santos Afonso, E., Rameix-Welti, M.-A., van der Werf, S. \& Naffakh, N. Host-range determinants on the PB2 protein of influenza A viruses control the interaction between the viral polymerase and nucleoprotein in human cells. Virology 362, 271-282 (2007).

175. Martin, K. \& Helenius, A. Nuclear transport of influenza virus ribonucleoproteins: the viral matrix protein (M1) promotes export and inhibits import. Cell 67, 117-130 (1991).

176. Stauffer, S. et al. Stepwise priming by acidic $\mathrm{pH}$ and a high $\mathrm{K}^{+}$concentration is required for efficient uncoating of influenza A virus cores after penetration. J. Virol. 88, 13029-13046 (2014).

177. Engelhardt, O. G Smith, M \& Fodor, E. Association of the influenza A virus RNA-dependent RNA polymerase with cellular RNA polymerase II. J. Virol. 79, 5812-5818 (2005).

178. Neumann, G., Hughes, M. T. \& Kawaoka, Y. Influenza A virus NS2 protein mediates vRNP nuclear export through NES-independent interaction with hCRM1. EMBO J. 19, 6751-6758 (2000).

179. Palese, P., Tobita, K., Ueda, M. \& Compans, R. W. Characterization of temperature sensitive influenza virus mutants defective in neuraminidase. Virology 61 , 397-410 (1974).

\section{Acknowledgements}

S.M.H. acknowledges grant 082098 from the Wellcome Trust and grants BB/K016164/1 and BB/F008309/1 from the Biotechnology and Biological Sciences Research Council. W.S.B. acknowledges grants 200187 and 205100 from the Wellcome Trust and grant BB/K002465/1 from the Biotechnology and Biological Sciences Research Council.

Author contributions

The authors contributed equally to all aspects of the article.

\section{Competing interests}

The authors declare no competing interests.

Publisher's note

Springer Nature remains neutral with regard to jurisdictional claims in published maps and institutional affiliations.

\section{Reviewer information}

Nature Reviews Microbiology thanks R. Fouchier, A. Mehle and other anonymous reviewer(s) for their contribution to the peer review of this work.

\section{RELATED LINKS}

FAO.org H7N9 situation update: http://www.fao.org/ag/ againfo/programmes/en/empres/H7N9/Situation_update. html 